\title{
Multifractal Analysis of Packed Swiss Cheese Cosmologies
}

\author{
J. R. Mureika \\ W. M. Keck Science Center, The Claremont Colleges, Claremont, \\ CA 91711 USA \\ Email: jmureika@jsd.claremont.edu \\ C. C. Dyer \\ Department of Astronomy and Astrophysics, University of Toronto, \\ Toronto, ON M5S $1 A 7$ Canada \\ PACS No. : Primary 98.65.-r ; Secondary: 95.30.Sf \\ Keywords: Cosmology - Large-scale structure of Universe, Gravitation, Relativity
}

\begin{abstract}
The multifractal spectrum of various three-dimensional representations of Packed Swiss Cheese cosmologies in open, closed, and flat spaces are measured, and it is determined that the curvature of the space does not alter the associated fractal structure. These results are compared to observational data and simulated models of large scale galaxy clustering, to assess the viability of the PSC as a candidate for such structure formation. It is found that the PSC dimension spectra do not match those of observation, and possible solutions to this discrepancy are offered, including accounting for potential luminosity biasing effects. Various random and uniform sets are also analyzed to provide insight into the meaning of the multifractal spectrum as it relates to the observed scaling behaviors.
\end{abstract}




\section{Do We Live in a Fractal Universe?}

The notion of a hierarchically-structured world is a recurrent theme in our understanding of Nature [1. According to the Cosmological Principle, the Universe must be homogeneous and isotropic. This oft cited-as-fact stipulation is the basis for the Friedmann-Robertson-Walker (FRW) solutions to Einstein's Field Equations, from which the expansion dynamics of the Universe are derived [2]. However, the breakdown of homogeneity (at least on smaller distance scales in the Universe) is quickly becoming an accepted ideal in cosmological circles. That is, while homogeneity requires matter to scale uniformly in space (i.e. $D_{F}=3$, equal probability scaling behavior in all spatial directions), actual measurements of the distributions suggest otherwise.

The existence of fractally-clustered matter was further emphasized by Peebles (see [3]), whose various two-point correlation analyses of three-dimensional catalogs yielded the exponent $\gamma \sim 1.7$, the co-dimension of which was taken to be the fractal dimension $D_{F}=3-\gamma \sim 1.3$. Some successive works confirmed this value, showing a study of the CfA redshift survey to agree statistically [4, 5]. However, these same studies cite model simulations with higher dimensionality, in particular $D \sim 2^{1}$.

Over the course of a decade, increasing evidence has been put forth to suggest that the dimensionality of a wide range of redshift survey catalogs yield such clustering dimensions. In the comprehensive publication [6], the results of fractal dimension analysis of numerous galactic catalogs are reviewed, with the general consensus that each data set reveals a unanimous $D_{F} \sim 2$ (see Table 1).

Since each catalog is limited in size and spatial extent (i.e. volume), the associated dimensions can only be statistically viable up to some effective radius $R_{\text {eff }}$ (the radius of the largest sphere one can inscribe in the associated sample, without surpassing the catalog boundaries). For most galaxy catalogs considered in the literature, these range up to $50 h^{-1} \mathrm{Mpc}$ (recall that $h^{-1}$ is the scaling factor in the Hubble Constant, $H_{0}=100 h \mathrm{~km} \mathrm{~s}^{-1} \mathrm{Mpc}^{-1}$ [6]). Pietronero et al. further extend the analysis to include clusters and superclusters, concluding that the two sets of data represent the same self-

\footnotetext{
${ }^{1}$ This discrepancy ultimately has its roots in the method of calculation. The dimension $D=D_{F}=2$ is the standard fractal dimension obtained by usual means, while The value $D \sim 1.3$, calculated from a two-point correlation function, is actually the $q=2$ multifractal dimension $D_{2}$. See Section 4
} 
similar structure at differing scales. While clusters are more distant, they are more luminous, and by counting the clusters as single objects, a coarser estimate of scaling is obtained over larger distances. This provides evidence for fractal behavior up to distances $1000 h^{-1} \mathrm{Mpc}$, with no suggestion that homogeneity ensues.

Of course, a potential limitation of such estimates is the lack of a definite third dimension. While angular spans may be accurately measured (barring external interference from sources such as gravitational lensing), the (co-moving) distance to the objects in question must rely on estimates from luminosity-distance relations or redshift measurements. These effects are introduced primarily by the recessional velocities of the objects, and in general may be obtained via application of the Hubble redshift law, whose simplest form for low redshifts is linear,

$$
r=\frac{c z}{H_{0}}
$$

and for general redshifts (Mattig's 1958 relation; see e.g. [6])

$$
r=\frac{c}{H_{0}} \frac{z q_{0}+\left(q_{0}-1\right)\left(\sqrt{2 z q_{0}+1}-1\right)}{q_{0}^{2}(1+z)},
$$

in an expanding Universe with deceleration parameter $q_{0}$. For $q_{0}=1 / 2$ (i.e. flat Universe), this can be shown to reduce to [6]

$$
r=6000\left(1-\frac{1}{\sqrt{1+z}}\right) h^{-1} \mathrm{Mpc}
$$

Typical redshifts for the majority of nearby catalogs are $z<0.05$, but deeper surveys such as ESP or LCRS can contain redshifts of the order $z \sim 0.2$ [7, 8]. Note that there is no correction for peculiar velocities in the associated figures of Table 1 [6].

The authors of [6] conclude that use of the Euclidean Hubble relation (11) in catalogs where the redshifts are high instead of (2) or (3) does not affect the estimated dimensionality, and thus the result $D_{F} \sim 2$ is stable to such variations. This claim is further elucidated in reference [9], who note that use of the Euclidean Hubble distance formula may be exported to distance greater than $600 \mathrm{Mpc}$ with no consideration of relativistic curvature effects ${ }^{2}$.

\footnotetext{
${ }^{2}$ In fact, this provides alternative support for the notion of "deformation independence" of the fractal dimension with respect to the embedding manifold, discussed in [10].
} 
Before proceeding, however, it should be emphasized that the aforementioned $D_{F} \sim 2$ fractal structure of the local Universe (with no transition to $D_{F}=3$ ) is a hotly debated subject, and is by no means to be interpreted as "fact". Rather, it is a movement largely spearheaded by the authors of [6], whom collectively have published over 50 related articles in the past 5 years. In fact, several earlier works 3 suggest that while the local structure may be fractal, the transition to homogeneity is clearly marked at about $5 h^{-1} \mathrm{Mpc}$. The general consensus tends to reflect this finding (i.e. that at least the local clustering structure can be described as a fractal), although the transition distance varies from catalog to catalog and analysis. More recently, the authors of reference [1] submit that there is no statistically or physically viable way to obtain a fractal scaling behavior for the ESP redshift survey and Abell clusters, instead citing a homogeneous $D \sim 3$ scaling, attributing the potential $D_{F} \sim 2$ to various redshift-related distance biases on the part of Pietronero et al., including the substitution of the Euclidean Hubble law in lieu of the FRW version! A rebuttal to this publication suggests that the cross-over in question is explicitly dependent on the cosmological model used for the calculations 12. Clearly, there is much disagreement in the literature, so one must be careful not to take each report at unquestioning face value.

As newer and more comprehensive redshift data becomes available from such current surveys as the Sloan Digital Sky Survey (SDSS) 13 or the 2dFGRS [14] (which are targeting well in excess of 100000 redshifts, and in the case of SDSS, 1000000 galaxies), the crux of this debate may be addressed with more certainty (or, on the other hand, such may serve to further complicate the issue!).

\section{The Packed Swiss Cheese Cosmological Model}

The notion of a fractal Universe defies the Cosmological Principle, which demands homogeneity and isotropy at all points. Locally, however, it is rather evident that the universe is not homogeneous. Any cosmological model which is used to represent the observed galaxy distribution must adhere to this point, lest its power of predictability be diminished.

Some of the earlier references to locally inhomogeneous cosmological models date back to Einstein and Strauss [15, as well as Schucking in the 1950s [15], and later Rees and Sciama in the 1960s [15]. Dubbed "Swiss Cheese" 
models, these constitute locally inhomogeneous but globally homogeneous spacetimes which everywhere satisfy Einstein's Field Equations. Further works studied the effects of multiple hole solutions vis-a-vis gravitational lensing effects with and without a Cosmological Constant [16, 17], and most recently the notion of optimally packed, volume-filling hole solutions, dubbed Packed Swiss Cheese cosmologies (PSC) [18, 19].

A sphere is inscribed in a zero-pressure, expanding FRW Universe of spatially-uniform density profile $\rho_{0}$, and the mass within is condensed to a smaller sphere of larger average density $\rho_{1}>\rho_{0}$. The total mass is conserved within the shell. Outside the inscribed radius, the space is still purely FRW, with line element

$$
\begin{aligned}
d s^{2} & =d t^{2}-R^{2}(t)\left[d \omega^{2}+S_{k}^{2}(\omega) d \Omega^{2}\right], \\
& =d t^{2}-R^{2}(t)\left[\frac{d r^{2}}{1-k r^{2}}+r^{2} d \Omega^{2}\right],
\end{aligned}
$$

for $\omega$ the angular radial coordinate, and the co-moving surface defined by $S_{k}(\omega)$ is either $\sin (\omega), \omega, \sinh (\omega)$ for positive, flat, or negative curvatures spaces respectively (equivalently one can write $S_{k}(\omega)=\sin (\sqrt{k} \omega) / \sqrt{k}$ for $k=+1,0,-1)$ [2, 17]. $d \Omega=d \theta^{2}+\sin ^{2}(\theta) d \phi^{2}$ is the standard (spherical) solid angle element. Hence, the CP is preserved on the exterior.

The metric of the vacuum interior of the hole is the Schwarzschild line element,

$$
d s^{2}=\left(1-\frac{2 G m}{r}-\frac{\Lambda r^{2}}{3}\right) d t^{2}-\frac{d r^{2}}{1-2 G m / r-\Lambda r^{2} / 3}-r^{2} d \Omega^{2},
$$

for general Cosmological Constant $\Lambda$ and interior mass $m$ (subject to appropriate matching conditions at the surface boundary [16]). If the internal mass density has some spatial extent, this can also possess an FRW line element (but not necessarily), although in the case of the PSC one considers only an interior point mass. Since their average density profile is unchanged, any number of holes may evolve independently of each other, since they have no gravitational influence on one another (provided the inscribed surfaces do not overlap) [17.

The PSC is conceptually similar to the classic "Apollonian Packing" problem of efficiently filling a region with tangential circles of varying sizes. In the former case, however, it is a three-dimensional packing problem with 
spheres in spaces of constant curvature. A point is chosen at random in a region of FRW space, as before, and a sphere of an arbitrarily large radius $R_{0}$ is inscribed. The mass within this sphere is shrunken down to a smaller radius $R_{0}^{\prime}$, increasing the local density and creating a density discontinuity at the boundary. A second point is chosen in the remaining continuous density region, and a second sphere is inscribed, subject to the constraint that it be tangential to the first. The interior mass is again contracted to a specified radius, and the process is repeated indefinitely. As mentioned, the contracted radius $R^{\prime} \rightarrow 0$.

Any number of packings and configurations can be obtained by varying the initial size and placement of the first sphere. Since there are no explicit scale constraints on the configurations, a fully recursive PSC can be formed by inserting within any sphere of one packing the contents of another. Such a model hearkens of self-similarity, at the very least on a statistical level. The libraries used for this study (see [19] for details) contain on average between 30000 to 90000 spheres (generally much larger than the size of the redshift surveys). Figure 1 shows a two-dimensional projection of a typical packing library.

There is no discussion herein of effective scales or cutoff radii, as with the survey catalogs, since the packing libraries are scaled in dimensionless units. Indeed, since one may recursively pack them at will, the overall physical scales may be set arbitrarily.

It should be noted that this Swiss Cheese model is different than that of Ribeiro [20, 21, 22, who also predicts a large-scale fractal structure. Comparisons with the aforementioned results are discussed in section 4.2

\subsection{Justification for a Swiss Cheese Cosmology}

The choice of a Swiss Cheese cosmology may seem at first to be without clear motivation, but it is in fact one of the most logical and consistent spacetimes in which to work. The Swiss Cheese solution is an exact solution of the Einstein Field equations at all the length scales involved. This is absolutely

necessary to begin consideration of fractal behavior since the very essence of the fractal approach is validity over a range of length scales.

The PSC model considered herein is built upon the Swiss Cheese solution, and while this solution at first appears to some to be artificial, it covers the essential field regions for an object embedded in a background universe. Thus it encompasses a near field, a medium field (i.e. the vacuum around the 
central object) and the far field, which just becomes the FRW background universe. The sharpness of the boundaries have little physical impact. Note that the sharp boundary of the Earth does not have any drastic effects when scene in terms of the gravitational potential (and thus the metric tensor either).

\subsection{Cosmological Principle, GR Style}

It is useful to re-formulate the Cosmological Principle in the language of General Relativity, insofar as the PSC models are concerned. Recall that the curvature of a manifold with metric $g_{a b}$ is defined via second derivatives of the metric, and this information is completely contained within the Riemann Tensor,

$$
R_{a b c d}=g_{a i}\left[\Gamma_{b d, c}^{i}-\Gamma_{b c, d}^{i}+\Gamma_{n c}^{i} \Gamma_{b d}^{n}-\Gamma_{n b}^{i} \Gamma_{c d}^{n}\right],
$$

(with $\Gamma_{j k}^{i}$ the associated Christoffel Symbols).

The Weyl Tensor in $n$ dimensions is [23]

$$
C_{a b c d}=R_{a b c d}+\frac{2}{n-2}\left(g_{a[d} R_{c] b}+g_{b[c} R_{d] a}\right)+\frac{2}{(n-1)(n-2)} R g_{a[c} g_{d] b} .
$$

where [ ] denotes the anti-symmetric sum with respect to index permutation. Since it can be shown that $C_{b a d}^{a}=0$, the Weyl Tensor is the trace-free portion of the Riemann Tensor, and complements the information contained within the Ricci Tensor (trace of Riemann). Combined, both objects contain the complete curvature information of the Riemann tensor, with Ricci representing the local curvature contributions (via Einsetin's equations, i.e. as a function of the local pressure $p$ and matter density $\rho$ via the energy-momentum tensor $T_{a b}=p g_{a b}+(\rho+p) u_{a} u_{b}$, with $u_{a}$ the associated four-velocity), and Weyl the non-local or external contributions.

A different interpretation is as follows. For a sphere of radius $r$ inscribed in a density field $\rho(r)$, the Weyl Tensor is a measure of the over/under-density of integrated (or enclosed) mass $\tilde{m}(r)$ to "average contained mass" $\bar{m}(r)$ That is, $C_{a b c d} \propto \tilde{m}(R)-\bar{m}(R)$, where

$$
\begin{aligned}
& \tilde{m}(R)=4 \pi \int_{0}^{R} \rho(r) r^{2} d r, \\
& \bar{m}(R)=(4 \pi / 3) R^{3} \rho(R),
\end{aligned}
$$


and $C_{a b c d}$ is evaluated on some closed surface which encloses the density field $\rho$ (see reference [16 for a complete derivation). If this quantity is nonzero, it signals an imbalance in the mass distribution, and hence provides a "gravitational compass". That is, by providing a preferred direction, it breaks the isotropy of the space. If, on the other hand, $C_{a i b j}$ vanishes on this surface, then the space contains no local tidal forces (no compass), and the Cosmological Principle is upheld if the mass distribution is homogeneous.

Every inscribed sphere in the PSC packings is itself such a surface (see Figure 11), and thus it is always possible to find a compound surface within the packing on which the Weyl Tensor vanishes. The space outside the inscribed boundary is FRW, by design, but that contained within the sphere is not. In the PSC model, it is assumed to be a vacuum, and thus has vanishing $T_{a b}$ (and thus vanishing Ricci Tensor).

\section{Measuring the Multifractal Spectrum of the PSC Models}

Effective scaling dimensions are obtained by the (three-dimensional) box counting method. Tables 24] show the calculated dimension $D_{q}, q=0,2$ (box and correlation) and $q \rightarrow \infty$, for several packings libraries of flat, positive, and negative curvatures respectively. Values of $D_{q}$ for $q<0$ are discussed in Section 6 ,

Covering cubes of side $d$ ranged roughly over two orders of magnitude, requiring 8 cubes at the largest scales, to about half the population size of the packings (i.e. about 1-2 particles per box on average). Below this limit, the box scales drop below the average interparticle distance, and the calculation becomes skewed by the finiteness of the data set. Since the libraries are themselves spherical distributions of points, then the largest box used in the counting is that which optimally fits within the spherical region, in order to avoid any spatial biasing of near-empty boxes in which there are few points (i.e. at the edges). This cubical subset accordingly contains a reduced fraction of the total library population as listed in the associated Tables, but

still provides for a decent statistical sampling of the distribution. Figures 24 demonstrate the box counting regression with associated confidence levels for the $q=0$, yielding the appropriate slopes. Note that " $q \rightarrow \infty$ " cannot be numerically realized, so the associated values cited herein correspond to the 
values of $D_{q}$ for $q \geq 60$, at which stage the estimates are observed to have reached a relatively stable value.

There is a fairly narrow range in the dimensions for each library, essentially yielding a possible range of dimensions between $D_{0}=2.5-2.8$, depending on the overall size of the sample and the choice of fit points, with correlation dimensions $D_{2}=2.4-2.6$ (see also Figures 11,13] for the general form of the $D_{q}$ spectra). The results might suggest that the positivelyand negatively-curved spaces yield slightly higher box dimensions than the flat case, although to within the cited fit error no definite determination may be made. In fact, to the accuracy of the fits, the Tables are virtually indistinguishable.

Note that $D_{q \rightarrow \infty}$ tends to approach a value near 2 in every case. This result has a simple geometric interpretation, and in fact provides a definite signature for the PSC mechanism. Since the $D_{q}$ values for large $q$ can be interpreted as local scaling dimensions, this implies the dense regions are effectively two-dimensional structures. This will be further discussed in Section 4, in comparison with reported multifractal indices for observational and simulated data.

Furthermore, based on the relative consistency of the dimensions, one could conclude that these are signatures of the construction algorithm, and independent of the space in which they are built. Note that the fractal dimension of two-dimensional Apollonian Packing is reported to be in the range $D_{F} \sim 1.31$ [24, thus perhaps the $D_{0} \sim 2.6-2.7$ is a signature of the 3-dimensional packing. This figure should be compared with that of Reference [25], which reports a value of $D_{F} \sim 2.4$ for 3-D Apollonian Packing (although the author of [26] notes this to be "crude"). This notion of construction mechanism identification will be further discussed later in this paper.

Based on conclusions drawn out in Reference [10, the dimensions in Tables 3 and 4 are obtained via box counting in the flat projective subspace. This projection is justified, however, since the overall angular extent is $2 \omega_{\max }=\pi / 5$ (see Section 5.1), which constitutes a rather small portion of the hyper/pseudospheres. It has been shown in [10] that curvature effects become minimal at about this angular extent. So, in some sense, one cannot expect to see any signs of curvature for such a limited library.

It should be noted that since the libraries represent only the first-level in the recursion, the depth ranges only to less than 3 orders of magnitude, beyond which point the scale approaches the size of the smallest spheres 
and/or the mean interparticle distance. Adding a second level of recursion could help to better define the overall dimension, although this can lead to significant increases in the computation time required to perform the operation. Future faster processors combined with efficiently-written algorithms will certainly be able to handle such a task. This work is currently underway by C. C. D. et al.

From a formulaic approach, it is interesting to note that homogeneity is preserved on the first scales of iteration in the box counting procedure. That is, cubes of side $\sim d, d / 2, d / 4$ (where $d \sim \pi / 10$ is the radius of the set) completely cover the distribution, giving counts of 8,64 , and (approximately) 256 respectively. This "space-filling" quality of the packings is consistent with the design of the Swiss Cheese cosmology (i.e. intended to satisfy the Cosmological Principle on the largest of scales).

As a quick check, these statistics are in relative agreement with fractal dimension calculations via alternative codes or methods. A shareware piece of software known as fd3 was obtained [27, which calculates the box dimension of an N-dimensional array of an arbitrary number of coordinates. The corresponding box-counting algorithm is based on the method of reference [28]. The fd3 software rather consistently pegs the dimension of the packings at $D_{0} \sim 2.5-2.7$, with mild variation in the correlation dimension $D_{2} \sim 2.4-2.6$, in rather good agreement with the above results.

\section{Comparison to Observational and Simulated Data}

The results of the previous section suggest that the reported $D_{F} \sim 2$ of largescale clustering (Table 11) cannot be reproduced by the PSC models with any level of certainty. This could imply either that the PSC model under consideration is limited in its predictive power, or equally that the cited $D_{F} \sim 2$ is incorrect. It may be that sample size plays a critical role here: the actual surveys have considerably smaller counts than the PSC models considered herein. Note that smaller models showed lower fractal dimensions, so while this could indicate different scaling estimates at lower ranges, it does not support the extension to larger scales. It is not unreasonable, then, to expect different scaling behavior over different ranges.

Various multifractal analyses of the available redshift catalogs have been 
performed, but unfortunately, there is a relative lack of these clustering analyses in the literature, as compared to the more traditional correlation function (single fractal dimension) investigations. However, the majority of those which can be found tend to indicate commonalities in the data (with the exception of studies such as those discussed previously in [11, although this was not a full multifractal analysis). Recall that values of $D$ obtained from the standard correlation analyses should generally correspond to $q=2$ (not $q=0$ ), although in some cases this tends to be a point of confusion among some authors and the related interpretations of analysis data. Analyses which are purely monofractal (correlation or conditional density) quote only a single exponent with no regard for the $q$-index, which makes for sometimes ambiguous cross-method comparisons. It was first noted in 29] that from a multifractal analysis of the CfA cluster, there existed a mismatch between the box dimension $\left(D_{0} \sim 2.1\right)$ and the widely reported correlation dimension $\left(D_{2} \sim 1.3\right)$, indicating that the large-scale clustering was inadequately described by a single scaling dimension.

Further investigations of CfA1 were reported in 4, 5, in which it was determined that the spectrum of generalized dimensions ranged from $D_{0} \sim 2$ to $D_{\infty} \sim 0.6-0.7$. More recently, multifractal dimensions were extracted for the CfA2 survey [30, 31], as well as the Las Campañas survey [31] (see references therein for details on each catalog). Again, a similar trend in dimensions was observed, although instead citing correlation dimensions with values $D_{2} \sim 2$ for $r \sim 5-30 \mathrm{Mpc}$, and strong local clustering behavior of $D_{\infty} \sim 1$ (noting that the discrepancy in $D_{2}$ values from those reported previously may be due to the spatial extent of the catalog considered [31]). It was further noted in 31] that the multifractal behavior was only observed over the aforementioned distance scales, with $D \sim 3$ beyond (i.e. a transition to homogeneity ensues, again contrary to "no transition" conclusions of [6]).

Similarly, cold dark matter (CDM) N-body simulations of gravitational collapse have also been studied, with essentially similar conclusions being drawn. These are of particular interest due to their natural connection to hierarchical clustering growth from small initial mass/density perturbations in the early Universe. The main difference, however, is that while observational data tend to show "fluid" multifractal structure, the latter simulations seem to display moreover a "bi-fractal" scaling [32, 33, 34, 35. Reference [32, presents a comprehensive set of dimension estimates for such simulations at various epochs (i.e. varying cosmological scale factor), as well for increasing biasing levels (the biasing level implies that clusters will only form for 
peak densities above a certain threshold value, generally a multiple of the standard deviation of the mass fluctuation [36]). The authors demonstrate that any number of box dimensions between $1.2-2.7$ are allowable, depending on the epoch and biasing levels (i.e. sensitivity to higher density fluctuations). Higher dimensions are observed at earlier epochs (when the matter distribution is still predominantly homogeneous), with the extreme lower dimensions occurring for high biasing. For all simulations considered, however, a consistent signature of the evolution is $D_{q} \rightarrow 1$ for larger $q$, due to the gravitational in the overdense clustering regions (i.e. non-linear density perturbation regime; see e.g. [3, 36] for a review).

References [34, 35] support this claim for behavior in various CDM Nbody simulations, where the high dimension $D \sim 2$ corresponds to largerscale correlations, with $D \sim 1$ behavior at smaller scales (and no interim scaling). This motivates debate as to how well even the N-body simulations reproduce observation. In reference [33, which studies the reliability of various fractal dimension estimation methods, the authors discuss several random process simulations which reproduce both the multifractal spectrum of observation, as well as the bi-fractal nature of such N-body simulations.

Thus, in addition to the mismatch between low $q>0$ dimensions, a further striking difference between the PSC models and the above cited results from both observational and simulated data are in the $D_{q}$ values for $q \rightarrow \infty$. This is attributed again to strong gravitational clustering effects at small scales which dominate the densest portions of the structures [33, 32, whereas the dimensions obtained in the packing library analyses herein tend to indicate that $D_{q} \sim 2$ for large $q$. The discrepancy is undoubtedly due again to the formation mechanism of the structure in question. The N-body simulations (and similarly the real local clustering effects) are evolutionary and dynamic, and hence their overall structure depends on the integrated gravitational interactions between the individual particles coupled with the scale expansion of the Universe. Thus, the densest structures will be "stringy", with one preferred direction being singled out. This is consistent with the observation of galactic "void-filament" structures, i.e. linear aggregate structures which cluster on surfaces.

The PSC algorithm, in contrast, creates the structure "on-the-fly", with no consideration of gravitational interactions. Again, its construction paradigm is purely geometric, based on volume-optimization, and the strongest clustering will occur by smaller spheres on the surfaces of the largest spheres. So, $D_{\infty} \sim 2$ can be interpreted as the densest regions clustering on surfaces, 
or having clustering behavior that has only two preferred directions. This is certainly a reasonable statement, since by the very nature of the packing routine, one should expect the smallest spheres to cluster on or near the surfaces of the largest inscribed spheres (one can obtain a sense of this from Figure 11). These dense regions, on the other hand, are reminiscent of the Great Wall and similar structures observed in such redshift surveys as CfA (see e.g. [37]).

As a quick clarification, the use of the term "preferred" in this case is used in an opposite fashion to the usual discussions of homogeneity. Note that $D_{F}=3$ would be described as structure having three "preferred" scaling directions (i.e. instead of no preferred direction - simply put, all directions are equally preferential).

The obvious mismatch in $D_{q}$ spectra between PSC and observation/simulation is discouraging, but it is not to say that the PSC model is inherently flawed. Rather, these figures should be seen as accepted limitations to the procedure, considering the accuracy of the data and associated statistics. The PSC models implicitly preserve the Cosmological Principle and Weyl flatness, while N-body simulations actively break these symmetries (or at the very least, do not concern themselves with consistent maintenance of this condition throughout the evolutionary process).

It is perfectly reasonable, however, to suggest that the two differing models are complementary, rather than in opposition. PSC shows large-scale homogeneity, preserves the CP, and displays strong clustering in two dimensions surrounding voids. All that is really missing is the filamentary structure. That is, the N-body simulations could always take place within any particular sphere of the packing. Recall that although the Weyl condition is satisfied on the large scale, in accordance with the FRW dust solutions, but locally (within the sphere) it can be violated (i.e. the vacuum is Ricci flat, but a non-vanishing Weyl is required to maintain overall curvature). Thus, the Weyl constraint provides a necessary boundary condition for the preservation of the CP. A hybrid of different clustering paradigms could help to yield a more realistic approximation to the observed structures and theoretical constructs. Equivalently, if the N-body simulations alone are truly bi-fractal, then combined with a PSC-like structure, one can obtain a more realistic model with which to compare observational data.

The fact that this discrepancy between dynamical and "instantaneous" formation paradigms can be readily signed through the multifractal analysis, and that the Universe more closely resembles the N-body data, can lend a 
sense of relief to philosophers. That is, this is a sure signal that the Universe has been evolving, or at the very least, was not created on-the-fly in the manner of the PSC! Thus, again, it would be interesting to study the multifractal scaling behavior of an N-body simulation nested within a PSC library, left to evolve over time. If this were to match observational data, the result could have profound impact for early Universe dynamics (not to mention the philosophical implications of a pre-determined structure in the beginning).

Along the same lines, perhaps the observational $D \sim 2$ of the galaxy clusters is actually a measure of this quantity, constrained by the relatively small sample space of candidate galaxies with respect to the estimated size of the Universe. Hence, the PSC could conceivably represent a "large-large" scale structure of the Universe, that is, much larger than the current observational limits. This would then be consistent with the above characteristics of observation and theory.

A brief mention is in order for another earlier hierarchical cluster model of related interest, that of Soneira and Peebles [38. Within a volume of space, a sphere or radius $R$ is inscribed, and within that sphere are placed $n$ spheres of radius $R / \rho$. In turn, within each of those spheres are placed $n$ new spheres of radius $R / \rho^{2}$, and so forth. It is interesting to note the conceptual similarities between this model and the SC packings, both of which are based on instantaneous positioning of spheres in a volume, and not on any time-evolution paradigm. The Soneira-Peebles model is not explicitly multifractal, but rather a simple monofractal of dimension $\log (n) / \log (\rho)$. The corresponding multifractal spectrum is effectively flat, such that $D_{q}=D_{F} \forall q$ for $r \in\left(R / \rho^{n-1}, R\right)$, where $n$ is the recursion level [5]. This, however, bears little resemblance to the PSC spectra, nor for that matter to observational data.

\subsection{Comparison with Random/Ordered Distributions}

Since the results do not suggest any method of discrimination between the three classes of models, it is important to address the question of how the multifractal structure of the packings compare to those of other random distributions of points. As a test, several toy random spherical distributions were populated using the $\mathrm{C}$ library function drand48(), which on each call generates a pseudorandom floating point number in the range $[0,1)$. Since curvature plays minimal or no role in the resulting spectra, for conciseness 
comparisons will be made only to the flat libraries.

One set of distributions is designed to be completely randomized, such that each $(x, y, z)$ coordinate is generated randomly. A second, dubbed "linear-random", randomly generates $(r, \theta, \phi)$ coordinates in the usual range. A third represents a completely uniform spacing of points which fills the appropriate volume. These three test sets, along with a random packing library, are displayed in Figures 5] and [6. Since no apparent distinctions can be made between the flat, open, or closed universe distributions, only the flat library SCF3 will be addressed in this section.

The simple Box dimension $\left(D_{0}\right)$ shows mild differences between each set, and in certain cases fails to accurately distinguish between them. However, the larger $q$ values do indeed show large variations in the overall structures. In particular, note that the linear-random distribution gives a box dimension of $D_{q} \sim 2.8$, but rapidly decreases for $q>0$ to a value of $D_{q} \rightarrow 1$ for $q \rightarrow \infty$. This exemplifies the ability of the multifractal spectrum to pick out anomalous structural qualities. Although the coordinates $(r, \theta, \phi)$ are populated randomly, the volume element of the space is not. For randomly selected $(x, y, z)$, the volume element $d V=d x d y d z$ is itself purely random, while randomly selected $(r, \theta, \phi)$ coordinates will yield a radially-weighted volume element $d V=r^{2} \sin \theta d r d \theta d \phi$. The overall clumping will reflect this $r$ preference, and hence will appear "pseudolinear". Since each set fills the volume almost homogeneously, and as such "fools" the box counting algorithm into a misrepresentation of the entire structure. This LR set can be compared to the local behavior of the N-body simulation dimensions discussed in Section 4, both of which are dominated by purely radial (e.g. gravitational) clustering behaviors at small scales. In fact, it is interesting/amusing to note that for this set that the correlation dimension $D_{2} \sim 1.9$, further suggesting that this test data could be considered a potential candidate for large-scale galaxy clustering (although purely in jest; this behavior appears more bifractal, due to the quick decrease to $D_{q} \sim 1$, and most likely lacks additional structural features beyond the $D_{q}$ spectra).

The $D_{\infty}$ value signals the linearity of the densest clustered regions. The randomly populated $(x, y, z)$ space $(\mathrm{RD})$ is more reflective of a truly random sample, as indicated by the dimensionality. Note that ideally, the set should have $D_{q}=D_{0}=3 \forall q$, but the variance can be understood to be software and data set limited (the same would be true of UD, which is just a variant of the RD). 


\subsection{Ribeiro's Tolman Swiss Cheese Cosmology}

As previously mentioned, the structure of a Swiss Cheese-like cosmology is a natural choice if one adheres to the existence of local inhomogeneity while adhering to the Cosmological Principle. Similar solutions have in fact appeared in the literature before, perhaps one of the most thorough being the model presented in the series of papers by Ribeiro [20, 21, 22]. It is interesting to compare these results - which discuss a relativistic fractal cosmology - to those of the current paper. Ribeiro's model is very similar in structure to the present Swiss Cheese model considered herein, matching Tolman solutions with FRW dust solutions and integrating local density distributions along the past light cone to calculate the observed fractal dimensions. For a variety of classes of solutions, the author finds fractal dimensions which range between $D_{F}=1.3-1.7$ depending on the model type under consideration. (subject to the constraint of obeying the de Vaucouleurs' density power law). The interested reader is directed to the aforementioned citations for further reading.

\section{Curvature Considerations}

\subsection{Evaluation Along Geodesics in $k= \pm 1$ Spaces}

Ideally, one must be careful when evaluating radial distances in non-flat geometries. Although it has been argued that the curved geometries considered herein possess "essentially-flat" characteristics due to their small angular extent, it is worth a quick check to see if there might exist any overt differences between the flat box estimates and those which do consider curvature effects.

The spatial portion of the FRW metric (4) can be written as

$$
d \sigma^{2}=d \omega^{2}+S_{k}(\omega)^{2}\left[d \theta^{2}+\sin ^{2} \theta d \phi^{2}\right],
$$

where the Cartesian embedding coordinates for the corresponding hypersurfaces are 2]

$$
\begin{aligned}
x & =S_{k}(\omega) \sin (\theta) \cos (\phi) \\
y & =S_{k}(\omega) \sin (\theta) \sin (\phi) \\
z & =S_{k}(\omega) \cos (\theta) \\
w & =T_{k}(\omega)
\end{aligned}
$$


Here, $T_{k}(\omega)=\cos (\omega)$ for $k=+1(\cosh (\omega)$ for $k=-1)$, and the radius of curvature has been set as $R=1$.

Realistically, one must compute the geodesic distance between points in the embedded space, instead of the straightforward Euclidean distance. For the positive and negative spaces considered herein, Equation 14 is evaluated along angular geodesic distances $r=\delta$, which is extracted from the usual inner product in the space between vectors $\left(\omega_{1}, \theta_{1}, \phi_{1}\right)$ and $\left(\omega_{2}, \theta_{2}, \phi_{2}\right)$,

$$
\begin{aligned}
\cos \delta= & \cos \left(\omega_{1}\right) \cos \left(\omega_{2}\right)+\sin \left(\omega_{1}\right) \sin \left(\omega_{2}\right)\left[\sin \left(\theta_{1}\right) \sin \left(\theta_{2}\right) \cos \left(\phi_{1}-\phi_{2}\right)\right. \\
& \left.+\cos \left(\theta_{1}\right) \cos \left(\theta_{2}\right)\right],
\end{aligned}
$$

for $k=+1$. The analogous expression for $k=-1$ obtained by the usual replacement for $S_{k}(\omega)$ and the appropriate sign change,

$$
\begin{aligned}
\cosh \delta= & \cosh \left(\omega_{1}\right) \cosh \left(\omega_{2}\right)-\sinh \left(\omega_{1}\right) \sinh \left(\omega_{2}\right)\left[\sin \left(\theta_{1}\right) \sin \left(\theta_{2}\right) \cos \left(\phi_{1}-\phi_{2}\right)\right. \\
& \left.+\cos \left(\theta_{1}\right) \cos \left(\theta_{2}\right)\right],
\end{aligned}
$$

The scaling dimensions may then be evaluated with "bottom-up" estimation techniques along the geodesic. The conditional average density $\Gamma(r)$ of points in the set, defined as

$$
\Gamma(r)=\frac{1}{N} \sum_{i=1}^{N} \frac{1}{A_{i}^{s}(r)} \frac{d N_{i}(r)}{d r} \propto r^{D-3},
$$

estimates the average change in number of points within a spherical shell of radius $r=\delta$ (area $A^{s}(r)$ ). The shell area $A^{s}(r)$ will depend on the value of $k$, however these effects are exceedingly small and can be ignored. This is reported to be a better estimation tool than the standard correlation function $\xi(r)=\left(r / r_{0}\right)^{D-3}-1$ (where $D_{F}=D_{2}$ ), which makes a priori assumptions of homogeneity with regard to the set under consideration (for an inhomogeneous fractal object can lead to spurious results) 6]. Of course, disagreements between dimension estimates from these two methods can also arise if the distribution is not a pure monofractal. If the set in question is fractal, then the number $N(r) \propto r^{D}$, and thus $\Gamma(r) \propto r^{D-3}$, which provides a good measure of the fractal dimension $D_{0}$ (and is frequently used in the cited references). Evaluating (13) over $N \sim 2000$ points per set or less, with an equivalent $R_{\text {eff }}<0.10$, one can obtain figures in good agreement with the cite box counting estimates. 
The plots of Figures 10 show $\Gamma(r)$ for sample libraries of each curvature, as compared with a base slope of 2.8. The fit itself is somewhat dependent on the choice of points, but all seem to suggest a similar slope. The smaller population sets show higher variability in the convergence of points to linearity, but this effect is smoothed out as the set grows in size. Note that the flat libraries tend to yield slightly smoother trends than the positive or negative sets. While this could be a curvature effect, it is unwise to make such an assertion without further investigation. The apparent non-linearity of each set below $r \sim 0.01$ is a recognized artifact of finiteness in volume-limited samples such as these, as the radial distances drop below the range of statisticallysignificant clustering 41. Hence, the cited dimensions are obtained for the linearity which ensues to the right of this "peak" This method is useful as a self-check here, since the calculated dimensions tend to support those obtained via the box counting method. Since it cannot be used to measure additional multifractal structure, however, its uses have been exhausted.

\section{$6 D_{q}$ for $q<0$}

The value of the parameter $q$ need not be restricted to positive integers, nor in fact need it be restricted to integers. The $D_{q}$ are well-defined for all $q \in \mathcal{R}$. Whereas the $D_{q}$ values for $q>0$ represent the scaling behavior of increasingly dense regions, those for $q<0$ correspond to the scaling of under-dense regions. Both the analyses of observational data and N-body simulations mentioned in Section 4 suggest that $D_{q} \rightarrow 3$ for $q \rightarrow \infty$, indicating that in all cases the least populous or dense regions scale effectively as homogeneous distributions.

Numerically, obtaining $D_{q}$ values of $q<0$ can be a very difficult quantity to estimate via box counting, since calculations becomes severely dependent on the finite size and population of the data set 33. Such behavior is observed herein - the associated error estimates grow significantly for $q \geq 0$, particularly for values of $q<-1.5$ or so. For example, the box counting algorithm yields $D_{q} \geq 2.7$ (0.3) for $q=-2$ for SCF3. Similarly, SCP5 shows $D_{q} \sim 2.8(0.2)$, and 2.7 (0.3) for SCN3. The other libraries considered demonstrate roughly similar behavior, although below these values of $q$, the fit errors quickly grow. In fact, in certain cases the estimated $D_{q}$ values begin to decrease for sufficiently small $q$, but with significantly increasing error (for all points fit). This could indicate that accurate determination of such gen- 
eralized dimensions is extremely dependent on the choice of points returned from the calculation.

There are several other means by which one can more accurately compute these values. A common method used in astrophysical analyses is the density reconstruction algorithm (see e.g. [5, 32] and related references), which determines the minimal radius $r(p)$ around a point for which the probability is $p$ of finding $p N_{\text {tot }}$ points. The corresponding partition function

$$
W(\tau, p)=\frac{1}{N_{\text {tot }}} \sum_{i=1}^{N_{\text {tot }}} r_{i}(p)^{-\tau} \propto p^{1-q},
$$

Recall that the multifractal spectrum $\left\{D_{q}\right\}$ is defined by the relation $\tau \equiv$ $\tau(q)=(q-1) D_{q}$, thus the corresponding generalized dimensions may be obtained accordingly. This method is claimed to converge well for $q \leq-1$, but is still applicable for a small range of positive $q$.

The calculation is somewhat computationally intensive, since ideally the radial calculations must be made for every point in the set. Generally, a smaller (random) sampling of the set can be used to evaluate the partition function. Sample calculations via implementation of this algorithm have been implemented for a range of probability values $p \in[0.01,0.1]$ (similar to the range used in [5]), for points whose inscribed radius is not overly large (else the calculation becomes saturated and useless), which generally includes up to several thousand points. In the cases of $k= \pm 1$, these are evaluated out along the geodesics (although as has been established, this complication likely isn't necessary).

Figures 11] 13 show sample $D_{q}$ plots for various libraries considered herein. The curves are shown to demonstrate the general trend in $D_{q}$ values, since there is sometimes a mild mismatch between the two methods (although within the box counting error). Thus, explicit points and error bars have been suppressed for the time being. The errors are roughly 0.1 , and so the curve can be assumed to be within $1-$ or $2-\sigma$ of the actual values. Initial results indicate that the scaling dimensions of the packings for small $q<<0$ approach $D_{q} \sim 3.0$, as was suggested by the box counting method. The rate at which the values for individual sets in each class (flat, positive, negative) approach the limiting value show some mild variation, but like the cases for positive $q$, this behavior could simply be due to the size of the data set under consideration (e.g. SCF1 shows markedly lower values than the rest, perhaps due to its smaller population size). 
The density reconstruction method is also applicable to (small) values of $q>0$, and it is noted that this provides good agreement with the box dimension estimates of Section 3. In particular, the estimates for $q \sim 2$ tend to be closer in agreement to the fits of Tables 2, 4 to within the cited error, thus providing yet another self-check for the estimates therein.

In certain cases, the $D_{q}$ value does not rise much above that of $D_{0}$, however it is uncertain whether or not this is an actual artifact of the data set, or rather calculation anomalies. This does not appear to be an explicit artifact of curvature, since it occurs for packings belonging to each class. It could be that these values of $D_{q}$ can serve as some variety of identification for varying initial conditions or the general distribution of sphere sizes (see [19]). That is, while $q>0$ is a measure of the strongest clustering, which can be interpreted as an overabundance of local mass at small scales, $q<0$ is to a certain extent a measure of open space, which certainly could vary from library to library. It could also be some manifestation of curvature on the construction paradigm at the largest levels [10]. Certainly, further investigation into the behavior is ideally warranted.

Coupled with the box counting results from before, these figures seem to consistently suggest an asymptotic value approaching $D_{q \rightarrow-\infty} \sim 3$. Such behavior has the simple interpretation that the regions of least clumping scale homogeneously, and thus no explicit structural information can be extracted from these values. Similar behavior is reportedly observed for the N-body simulations discussed previously, as well as the analysis of observational data from the diverse galaxy catalogs. Thus, differentiation of formation models seems to become even more ambiguous for this range of scaling. The strong clustering behavior for $q \gg 0$ gives a much more intuitive and exploratory glimpse of the inherent structure qualities of the set.

\section{Biasing Counts by Luminosity / Mass}

As previously noted, the estimated scaling dimensions for the packing libraries are high due to the density of points per allocated volume. Another issue is the unbiased nature of the counts. Simply put, the libraries are geometric configurations which satisfy the relativistic field equations. Each point is assigned a mass, which is a function of the inscribed radius within the constant density field, dependent on the curvature of the spatial manifold. However, to date this value has not been factored into the counting 
scheme.

The observed three-dimensional distributions are interpolated from measured catalogs - what one sees is what one gets. However, it is not unreasonable to suggest that what one sees is not what is actually there! The notion of "missing mass" is a recurring theme in many cosmological endeavors, ranging from dark matter issues, to low luminosity objects. Furthermore, it is common-place in observational astronomy to simply excluded objects whose luminosity is below a given threshold, irrespective of whether or not it is visible. If it does not meet the selection criteria for the catalog in question, it is omitted altogether. Furthermore, the issue of luminosity biasing or segregation effects is one which is frequently raised in the debate surrounding the $D \sim 2$ over all length scales. For example, it has been suggested that luminosity segregation favors brighter galaxies at greater distances, which consequently implies a biasing toward stronger clustering, which could skew the actual measured dimension from homogeneity to some different scale (see [32] and associated references therein).

The mass-to-light ratio for galaxies is somewhat dependent on factors such as age, morphological type, and so forth. It seems reasonable to impose a mass cutoff in the packing libraries as a first-pass gauge of this effect. That is, can one impose constraints on the range of lower mass cutoffs from which one can extract a dimension of $D \sim 2$ for the packing libraries? For most galaxies of a particular class, this ratio is roughly a constant, i.e. $M(L) \propto L^{\beta}, \beta \sim 1$. For simplicity, such a relation will be assumed for the analysis herein, and thus the results can provide a good "boundary" for what one might expect in realistic observational circumstances. Since the initial matter field is assumed to be of constant density $\rho_{0}$, the mass $M_{i}$ of the $i^{\text {th }}$ sphere may be calculated by the usual relations

$$
\begin{gathered}
M_{i}=\frac{4 \pi \rho_{0}}{3} R_{i}^{3}, \\
M_{i}=\pi \rho_{0}\left(2 R_{i}-\sin \left(2 R_{i}\right)\right), \\
M_{i}=\pi \rho_{0}\left(\sinh \left(2 R_{i}\right)-2 R_{i}\right),
\end{gathered}
$$

where $R_{i}$ is the inscribed (angular) radius of the sphere, for flat (15), positive (16), and negative curvatures (17). The latter expressions are obtained by integrating the FRW volume element for the respective values of $k$. Since $\rho_{0}$ is arbitrary, one can set $\rho_{0}=1$ WLOG.

Figure 15 shows the mass-reduced $D_{q}$ spectra for various reduced libraries, using a mass cutoff approximately $0.01 \%$ that of the largest sphere mass. In 
terms of the assumed mass-to-light ratio, this logically amounts to a similar scaling for the object's luminosity. The reduced data sets contain roughly $10 \%$ of the original number, but still accounts for over $90 \%$ of the total enclosed mass. Similar results are obtained for the other packing libraries using a similar cutoff. This indicates that the majority of the spheres are relatively small in mass, and thus by the mass-to-light ratio, would most likely be much fainter relative to the remaining points. Hence, the smaller masses serve to "smooth out" the overall matter distribution, leading to the saturation of homogeneity observed in the upper-most levels of the box counts.

Note the somewhat wide variation in limiting values of $D_{q}$ for negative $q$. Although the data confirms sparser clustering in these regimes, the asymptotic value which $D_{q}$ assumes tends to be somewhat dependent on the library. Initial results seem to suggest that there is no preferential pattern for specific geometries, with both possessing slow and fast approaches to a higher limiting dimension. It may be the case, as discussed previously, that further investigation into the $q<0$ regime could potentially shed light on the associated geometry, although a better understanding of the sensitivity of the method to population sizes is required. The authors of 32 similarly suggest that $q<0$ values could help discriminate between differing initial power spectrum perturbation conditions for N-body simulations.

Table 5 displays the results for the associated cutoffs for each the libraries of Tables 2, 4. Again, note that there is no explicit signature variation in the estimates between curvature cases. The SCP libraries show slightly higher $D_{0}$ and lower $D_{\infty}$ values than the SCF and SCN packings, albeit all equal to within the associated error. The same mass cutoff has been applied to each case, even though there is mild variation in the overall masses for each library (depending on the packing number). However, this is most likely a small consideration which does not significantly affect the end result. This variation could also be due to volume limitation constraints which exist in the closed manifold, but not in the flat and open cases.

Thus, it would appear that the use of luminosity cutoff can potentially bring the box and correlation dimensions of the libraries in closer agreement to the reported values of reference [6] (albeit with larger fit error), which suggests that such a biasing mechanism could help to rationalize the aforementioned discrepancy between the PSC models and observation (if correct). Such luminosity biasing in Abell clusters was discussed in detail by Bahcall and Soneira, who showed a mismatch in the galaxy and cluster spatial corre- 
lation lengths of at least a factor of 5 [39] as well as a strong dependence of the correlation function on cluster richness. The more recent analysis of the SSRS2 redshift survey presented in 40] further supports the conclusion that there exists a strong connection between statistical clustering and luminosity, in particular weighted toward bright galaxies (e.g. $M \leq-21$ ) over fainter ones. The authors comment that these results are largely inconsistent with current theoretical models, however this paper indicates that the PSC could offer a resolution to this observed effect over other clustering paradigms.

Furthermore, this can help support the notion that observed galaxies (luminous matter) can form a fractal distribution (subset) within a largely homogeneous matter distribution, much the same way the distribution of mountain peaks is fractal, while the Earth itself is largely spherical [42. Observationally, similar cutoff biasing has been recorded, and that the associated fractal dimensions tend to rise for a decreasing galaxy luminosity threshold [6, 42. Thus, the ideas presented herein are consistent with reported astrophysical procedures, and could always be used to help provide insight into similar "missing mass" investigations. As with the fully unbiased model, however, the values of $D_{\infty}$ for all libraries considered tend to cluster around $D_{\infty}=2$, which again does not match observational and N-body simulated models (whose $D_{\infty} \sim 1$, as previously discussed).

However, some caution must be exercised in interpreting such results. This trend may be moreover a statistical manifestation influenced by a different linear fit choice than an actual structural change in the set. In fact, by neglecting the smallest box size count in the fits for the higher cutoff biased sets, the $D_{0}$ values can be shown to rise to $\sim 2.4-2.5$ with smaller fit error, suggesting that the dimension is potentially higher than indicated in Table [5] (although still lower than the estimated $D_{0} \sim 2.7$ for the whole library). There is also a marginally larger range between the differing dimension estimate techniques for the mass-biased libraries. For example, the density reconstruction method tend to yield values of $D_{q} \sim 2.3-2.5$ or so for $q \sim 0$ (see the trends of Figure [15), indicating potential limitations of the box counting technique.

Figure 16]demonstrates the trend in dependence of the $D_{q}$ values on cutoff size, both for fixed number of iterations for all cutoff levels, as well as adjusted numbers for smaller set populations. The estimates remain largely unaffected until a cutoff of $>0.001 \%$ is reached (leaving about $20 \%$ of the spheres and $96 \%$ of the total contained mass), after which there is a relatively quick drop in $D_{0}$ with respect to $D_{\infty}$ for the fixed number of iterations. Whereas, 
adjusting the number of box levels (reducing by one) helps to correct the curve to a higher estimate. Beyond about $0.04 \%$, the dimensions approach roughly the same value $D<2.0$, suggesting a type of monofractal behavior. At this point the population of the set has been reduced to $2 \%$ of the original, thus the statistics could start to become skewed by finiteness effects due to a larger average point separation.

The box counting technique has at times been criticized in the literature for being too sensitive to discreteness effects, especially when the population size is small (causing spurious results or underestimates of the actual scaling dimensions) [34, 35]. In other cases, box counting is hailed as a quite robust and stable method for estimation of the associated statistics, and furthermore the density reconstruction method is claimed to give dimension overestimates for limited size sets [33. Thus, there is a certain level of disagreement and confusion in the community with respect to the utility of any of these methods.

So, when few data points are available such as in this situation, it is probably best to take some variety of average dimension as calculated by differing methods. Although the measured $D_{0}$ dimensions do drop when lower masses are discounted, the actual magnitude of the drop seems sensitive to the size of the data set (and measurement method). Whether or not this represents a skew in the measured dimension as a result of luminosity biasing is somewhat unclear. Thus, such biasing results are more susceptible to statistical anomalies (highly dependent on the choice of points in the box count fit) than are the full models, and care must be taken in their use. It is, however, worth noting that the $D_{\infty}$ values remain relatively consistent at $D_{\infty} \sim 2$, indicating a certain robustness of the data. This is a definite signature of the packing structure.

From a physical point of view, one must recall that the PSC models are merely weighted points. As mentioned in a previous section, one could imagine replacing each point with another type of set, such as an evolutionary N-body simulation, which could then conceivably yield finer clustering effects (and potentially yield a $D_{\infty} \sim 1$ behavior). If the dimensions of Table 5 are actually statistical properties of the luminosity-biased sets, then it could constitute a possible solution to reconciling the mismatch with observational data. 


\section{Future Considerations}

The basic premise of the packing algorithm is to ensure preservation of the Cosmological Principle via maintenance of Weyl flatness. Although this is done by compressing the matter contained within a spherical region, there is a priori no reason for this choice (apart from the motivation of gravitational collapse). Suppose instead the matter were expanded to lie along the spherical shell described by the inscription. This would create a thin sheet of matter, consistent with the two-dimensional structures complemented by voids, evident both from observation as well as the multifractal analysis. The matter could then be allowed to coalesce by some gravitational mechanism on the sheets, yielding the appropriate linear structures. Alternatively, the packing algorithm could randomly choose between collapse and expansion for the spheres, and in the case of a collapse, the interior matter is allowed to cluster via N-body behavior. Whether or not such models are physically realizable is unknown, but they could nevertheless provide yet another model from which to study and reconcile the observed large-scale structures in the Universe, and as mentioned, stand to uphold the Cosmological Principle on many levels.

Furthermore, the use of the monofractal dimension can frequently shortchange the characterization of a clustering set, since it is possible to have vastly differing structures which possess the same base dimension $D_{F}=D_{0}$. Although the multifractal spectrum can help differentiate such situations, a different consideration is the lacunarity of the set. This measure, often associated with the texture of a fractal, provides an estimate of the "voidness" (rather than "clumpiness") (see e.g. [1, 26, 43, 44]). Such an investigation is currently underway by J. R. M..

\section{Acknowledgments}

Funding for this work provided by the Natural Sciences and Engineering Research Council of Canada and the Walter C. Sumner Foundation. Thanks to Allen Attard for provision of the PSC libraries. 


\section{References}

[1] Mandelbrot, B. B. The Fractal Geometry of Nature, W. H. Freeman and co. (1983)

[2] Misner, C. W., Thorne, K. S., and Wheeler, J. A., Gravitation, W. H. Freeman and Company, New York (1973)

[3] Peebles, P. J. E., Principles of Physical Cosmology, Princeton University Press (1993)

[4] Jones, B. J. T. et al., Ap. J. 332, L1 (1988)

[5] Martinez, V. J. et al., Ap. J. 357, 50 (1990)

[6] Sylos Labini, F., Montuori, M., and Pietronero, L, Phys. Rep. 293, 61 (1998)

[7] Vettolani, G. it et al., Astron. Astrophys. 325, 954 (1997)

[8] Schectman, S. A. et al., Ap. J. 470, 172 (1996)

[9] Célérier, M. N., and Thieberger, R., Asrtron. Astrophys. 367, 449 (2001)

[10] Mureika, J. R., Topics in Multifractal Analysis of Two- and ThreeDimensional Structures in Spaces of Constant Curvature, Ph.D. Thesis, Graduate Department of Physics, University of Toronto (2001)

[11] Scaramella, R. et al., Astron. Astrophys. 334, 404 (1998)

[12] Joyce, M. et al., Astron. Astrophys. 344, 387 (1999)

[13] See Sloan Digital Sky Survey website, http: www.sdss.org/ and citations therein

[14] Colless M. M., Phil. Trans. Roy. Soc. Lond. A 357, 105 (1999)

[15] Einstein, A. and Strauss, E. G., Rev. Mod. Phys. 17, 120 (1945); Schücking, E. Z. Phys. 137, 595 (1954); Rees, M. J., and Scieama, D. W., Nature 217, 511 (10 Feb 1968)

[16] Dyer, C. C., Ph.D. Thesis, University of Toronto Department of Astronomy (1973) 
[17] Kantowski, R., Ap. J. 155, 89 (1969); Dyer, C. C. and Roeder, R. C., Ap. J. 189, 167 (1974); Dyer, C. C., Mon. Not. Roy. Ast. Soc. 175, 429 (1976); Dyer, C. C., in Theory and Observational Limits of Cosmology, 325, W. Stoeger (ed.), Vatican Observatory (1987); Dyer, C. C. and Oattes, L. M., Proc. Second Can. Conf. on General Relativity and Relativistic Astrophysics, 59, Coley, A., Dyer, C., and Tupper, B. (eds.), World Scientific Publishing, Singapore (1988) Dyer, C. C. and Ip, P. S. S., Proc. Second Can. Conf. on General Relativity and Relativistic Astrophysics", 272, Coley, A., Dyer, C., and Tupper, B. (eds.), World Scientific Publishing, Singapore (1988); Dyer, C. C. and Oattes, L. M., Ap. J. 326, 50 (1988); Dyer, C. C. and Ip, P. S. S., Mon. Not. Roy. Ast. Soc. 235, 895 (1988); Dyer, C. C. and Harper, J. F.,Proc. Third Can. Conf. on General Relativity and Relativistic Astrophysics, Tupper, B. (ed.), World Scientific Publishing, Singapore (1990)

[18] Dyer, C. C., personal communication

[19] Attard, A., "Simulating a Swiss-cheese Cosmological Model", University of Toronto Department of Astronomy and Astrophysics, M.Sc. project report (1997)

[20] Ribeiro, M. B., Ap. J. 388, 1 (1992)

[21] Ribeiro, M. B., Ap. J. 395, 29 (1992)

[22] Ribeiro, M. B., Ap. J. 415, 469 (1993)

[23] Hawking, S. W. and Ellis, G. F. R., The Large Scale Structure of SpaceTime, Cambridge University Press (1973)

[24] Manna, S. S., and Herrmann, H. J., J. Phys. A: Math. Gen. 24, L481 (1991)

[25] Boyd, D., Math. Computation 27, 369 (1973)

[26] Stern, I., "On Fractal Modeling in Astrophysics: The Effect of Lacunarity on the Convergence of Algorithms for Scaling Exponents", in Astronomical Data Analysis Software and Systems VI, Gareth Hunt and H. E. Payne (eds.), APS Conference Series 125 (1997) 
[27] Sarraille, J. J. and Myers, L. S., Educ. Psych. Meas. 54(1), 94 (1994); software available for download at ftp://www.cs.csustan.edu/pub/fd3/

[28] Liebovitch L., and Toth, T, Phys. Lett. A141, 386 (1989)

[29] Martinez, V. J. and Jones, B. J. T., Mon. Not. R. Astr. Soc. 242, 517 (1990)

[30] Kurokawa, T., Morikawa, M., and Mouri, H., Astron. Astrophys. 344, 1 (1999)

[31] Kurokawa, T., Morikawa, M., and Mouri, H., Astron. Astrophys. 370, 358 (2001)

[32] Valdarnini, R., Borgani, S., and Provenzale, A., Ap. J. 394, 422 (1992)

[33] Borgani, S. et al., Phys. Rev. E 47, 3879-3888 (1993)

[34] Dubrelle, B., and Lachièze-Rey, M., Astron. Astrophys. 289, 667 (1994)

[35] Colombi, S., Bouchet, F. R., and Schaeffer, R., Astron. Astrophys. 263, 1 (1992)

[36] Padmanabhan, T., Structure Formation in the Universe, Cambridge University Press (1993)

[37] Geller, M., and Huchra, J., Science 260, 1175 (1989)

[38] Soneira, R., and Peebles, P. J. E., Ap. J. 83, 845 (1978)

[39] Bahcall, N. and Soneira, R., Ap. J. 270, 20 (1983)

[40] Benoist, C. et al., Ap. J. 472, 452 (1996)

[41] Montuori, M., et al., Europhys. Lett. 39, 103 (1997)

[42] Durrer, R., and Sylos Labini, F., Astron. Astrophys. Lett. 339, L85 (1998)

[43] Durrer, R. et al., Eurpohys. Lett. 40, 491 (1997)

[44] Martínez, Vincent J. and Saar, Enn, "Clustering statistics in cosmology", to appear in Astronomical Data Analysis II, J.-L. Stark and F. Murtagh (eds), SPIE Proceedings 4847 (2002) 


\begin{tabular}{lcc}
\hline Survey & $D_{F}$ & Approx. Size \\
\hline CfA1 & $1.7(0.2)$ & 1800 \\
CfA2 & $\sim 2$ & 11000 \\
SSRS1 & $2.0(0.1)$ & 1700 \\
SSRS2 & $\sim 2$ & 3600 \\
LEDA & $2.1(0.2)$ & 75000 \\
IRAS 1.2/2 Jy & $2.2(0.2)$ & 5000 \\
Perseus-Pisces & $\sim 2.1$ & 3300 \\
ESP & $1.8(0.2)$ & 3600 \\
Las Campañas (LCRS) & $2.2(0.2)$ & 25000 \\
\hline
\end{tabular}

Table 1: Galaxy fractal dimension calculations for various redshift surveys (compiled from [6]). 


\begin{tabular}{cccc}
\hline Library (pts) & $D_{0}$ & $D_{2}$ & $D_{\infty}$ \\
\hline SCF1 (22076) & $2.5(0.1)$ & $2.4(0.1)$ & $2.2(0.1)$ \\
SCF2 (42325) & $2.6(0.1)$ & $2.6(0.1)$ & $2.2(0.1)$ \\
SCF3 (49797) & $2.7(0.1)$ & $2.6(0.1)$ & $2.2(<0.1)$ \\
SCF4 (73936) & $2.7(0.1)$ & $2.6(0.1)$ & $2.3(0.1)$ \\
SCF5 (82788) & $2.8(0.1)$ & $2.6(0.1)$ & $2.3(<0.1)$ \\
\hline
\end{tabular}

Table 2: Calculated dimensions for flat space packings. Cube size ranges from homogeneous saturation at largest scales (8 cubes) to roughly $2-4$ points per cube (ranging from scales $(\sim \pi / 10-\pi / 300))$. 


\begin{tabular}{cccc}
\hline Library (pts) & $D_{0}$ & $D_{2}$ & $D_{\infty}$ \\
\hline SCP1 (31904) & $2.6(0.1)$ & $2.5(0.1)$ & $2.2(0.1)$ \\
SCP2 (35268) & $2.7(0.1)$ & $2.6(0.1)$ & $2.2(0.1)$ \\
SCP3 (47140) & $2.7(0.1)$ & $2.6(0.1)$ & $2.2(0.1)$ \\
SCP4 (54966) & $2.7(0.1)$ & $2.6(0.1)$ & $2.2(0.1)$ \\
SCP5 (80437) & $2.8(0.1)$ & $2.6(0.1)$ & $2.3(0.1)$ \\
\hline
\end{tabular}

Table 3: Calculated dimensions for positively-curved space packings. 


\begin{tabular}{cccc}
\hline Library & $D_{0}$ & $D_{2}$ & $D_{\infty}$ \\
\hline SCN1 (32965) & $2.6(0.1)$ & $2.5(0.1)$ & $2.1(0.1)$ \\
SCN2 (49343) & $2.7(0.1)$ & $2.6(0.1)$ & $2.2(0.1)$ \\
SCN3 (60245) & $2.7(0.1)$ & $2.6(0.1)$ & $2.3(0.1)$ \\
SCN4 (65899) & $2.7(0.1)$ & $2.6(0.1)$ & $2.3(0.1)$ \\
SCN5 (83863) & $2.8(0.1)$ & $2.6(0.1)$ & $2.3(0.1)$ \\
\hline
\end{tabular}

Table 4: Calculated dimensions for negatively-curved space packings. 


\begin{tabular}{llcccc}
\hline Lib. & $\#$ (\% tot) & \% mass & $D_{0}$ & $D_{2}$ & $D_{\infty}$ \\
\hline SCF1 & $4526(20.5)$ & 96.0 & $2.1(0.3)$ & $2.1(0.2)$ & $1.9(0.1)$ \\
SCF2 & $5074(12.0)$ & 93.6 & $2.1(0.3)$ & $2.1(0.2)$ & $1.9(0.1)$ \\
SCF3 & $5218(10.5)$ & 92.8 & $2.2(0.3)$ & $2.2(0.2)$ & $1.9(0.1)$ \\
SCF4 & $5506(7.4)$ & 91.1 & $2.2(0.3)$ & $2.2(0.2)$ & $1.9(0.1)$ \\
SCF5 & $5599(6.7)$ & 90.6 & $2.2(0.3)$ & $2.2(0.2)$ & $1.9(0.1)$ \\
\hline SCP1 & $4744(14.9)$ & 94.6 & $2.1(0.3)$ & $2.1(0.2)$ & $1.8(0.2)$ \\
SCP2 & $5848(16.6)$ & 93.3 & $2.3(0.3)$ & $2.2(0.2)$ & $2.0(0.1)$ \\
SCP3 & $5108(10.8)$ & 92.9 & $2.2(0.3)$ & $2.2(0.2)$ & $2.0(0.1)$ \\
SCP4 & $5233(9.5)$ & 92.4 & $2.2(0.3)$ & $2.2(0.2)$ & $1.9(0.1)$ \\
SCP5 & $5542(6.9)$ & 90.6 & $2.2(0.3)$ & $2.2(0.2)$ & $1.8(0.2)$ \\
\hline SCN1 & $4993(15.1)$ & 94.7 & $2.1(0.3)$ & $2.1(0.2)$ & $1.9(0.1)$ \\
SCN2 & $5294(10.7)$ & 93.0 & $2.2(0.3)$ & $2.2(0.2)$ & $1.9(0.1)$ \\
SCN3 & $5409(9.0)$ & 92.1 & $2.2(0.3)$ & $2.2(0.2)$ & $1.9(0.1)$ \\
SCN4 & $5536(8.4)$ & 91.7 & $2.2(0.3)$ & $2.2(0.2)$ & $1.9(0.1)$ \\
SCN5 & $5837(7.0)$ & 90.6 & $2.2(0.3)$ & $2.2(0.2)$ & $2.0(0.2)$ \\
\hline
\end{tabular}

Table 5: Mass-reduced box counts for all libraries, showing box, correlation, and limiting $D_{q \rightarrow \infty}$ values. Lower $D_{0}$ values with higher errors may be artificially lower due to finiteness effects. 


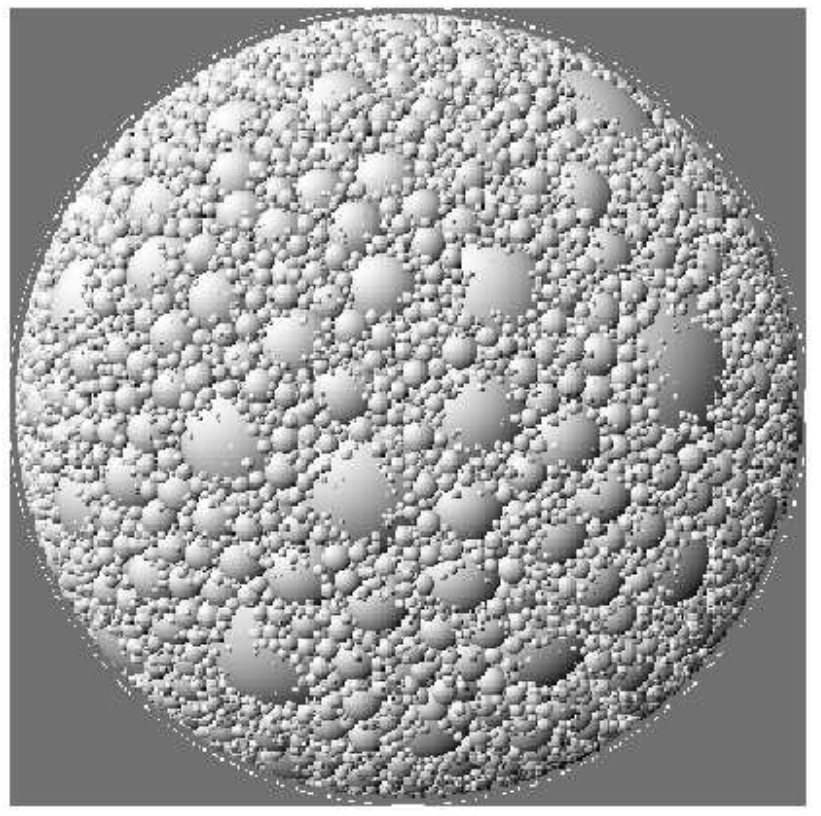

Figure 1: Sample projected two-dimensional visualization of packing library, containing approximately 35000 spheres. Surfaces represent the inscribed radius, and not the actual contained matter. 


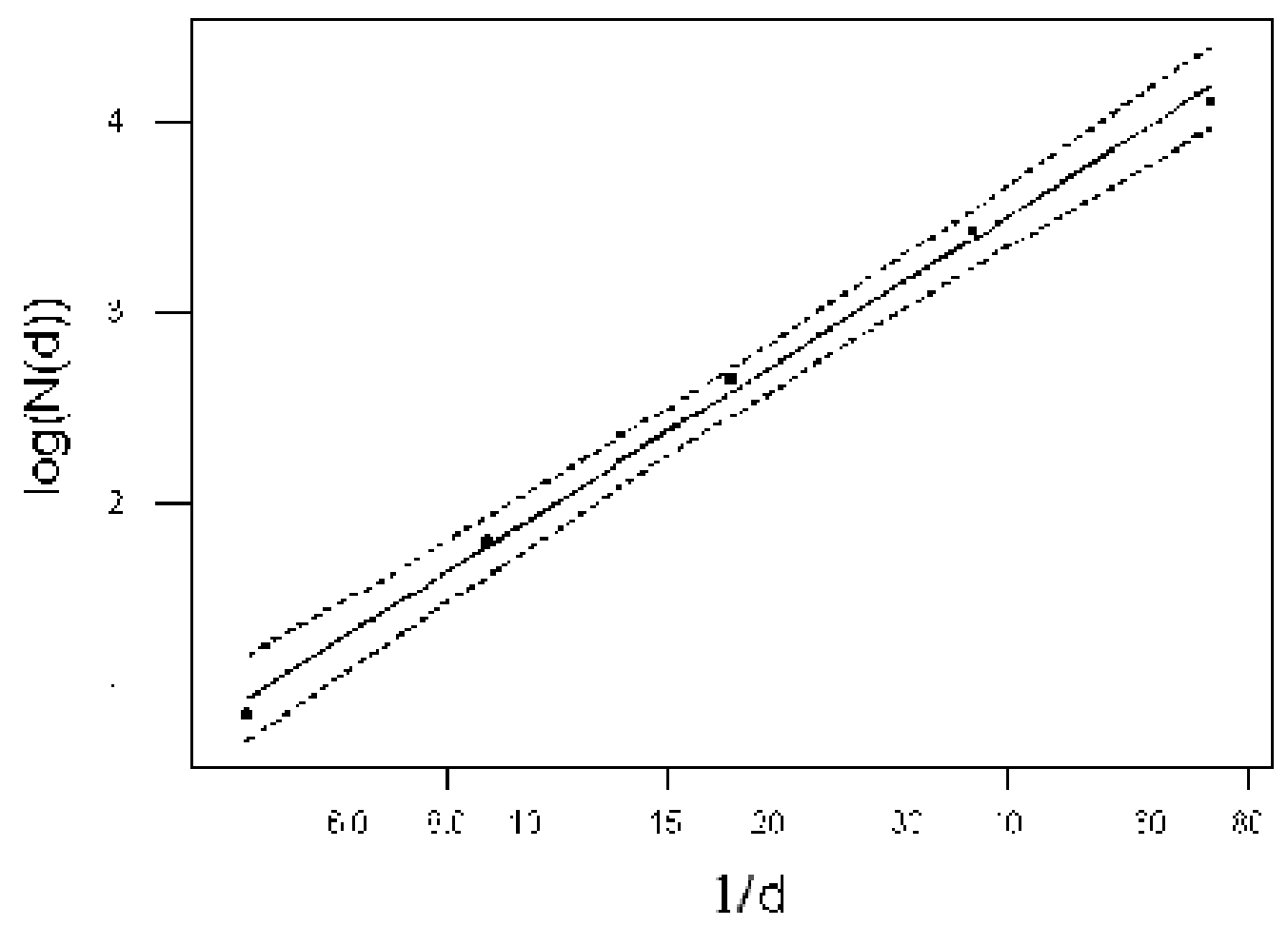

Figure 2: Box counting $\left(D_{0}\right)$ fit for SCF3, yielding $D_{0}=2.7(0.1)$. 


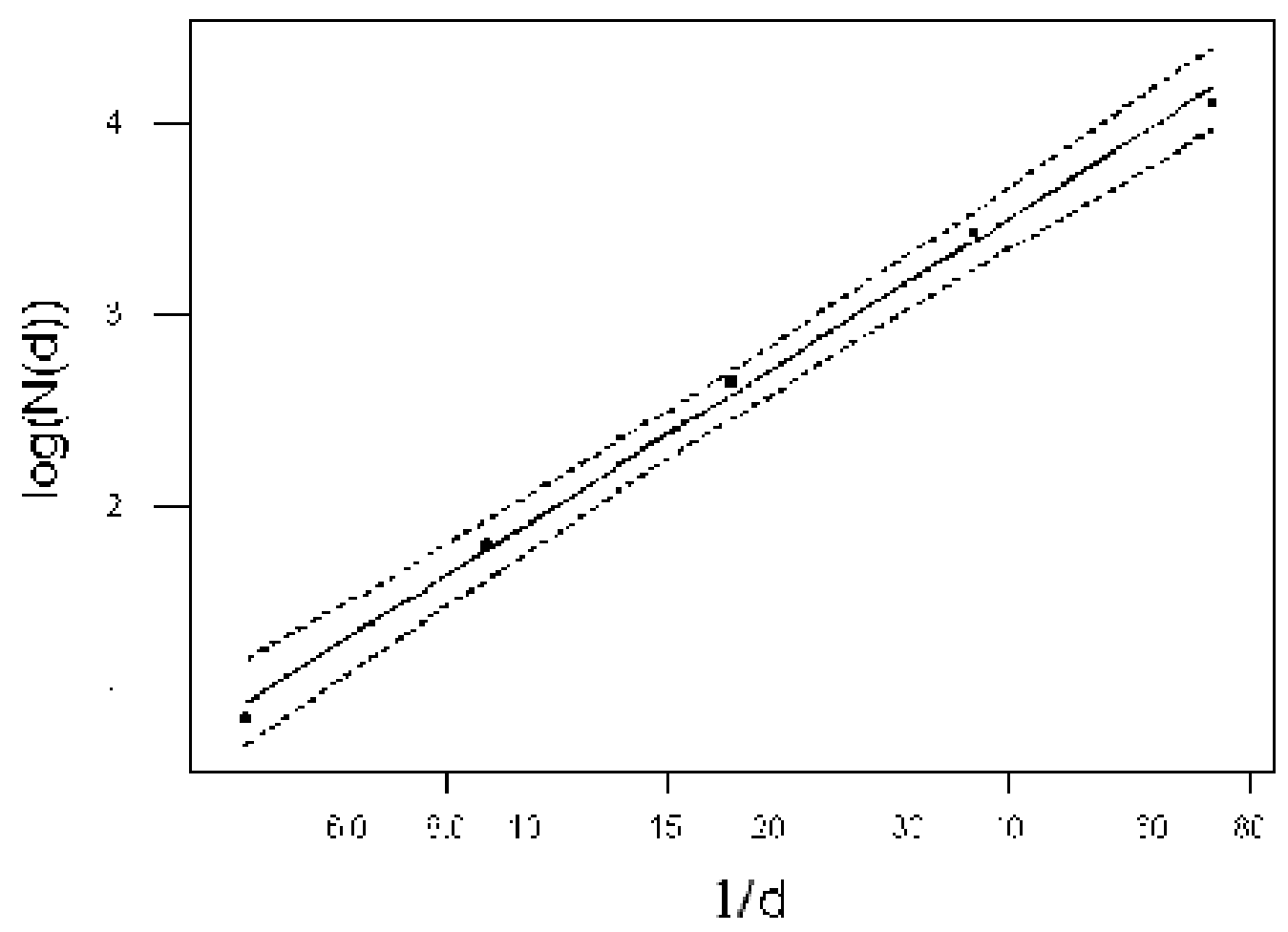

Figure 3: Box counting $\left(D_{0}\right)$ fit for SCP3 yielding $D_{0}=2.7(0.1)$. 


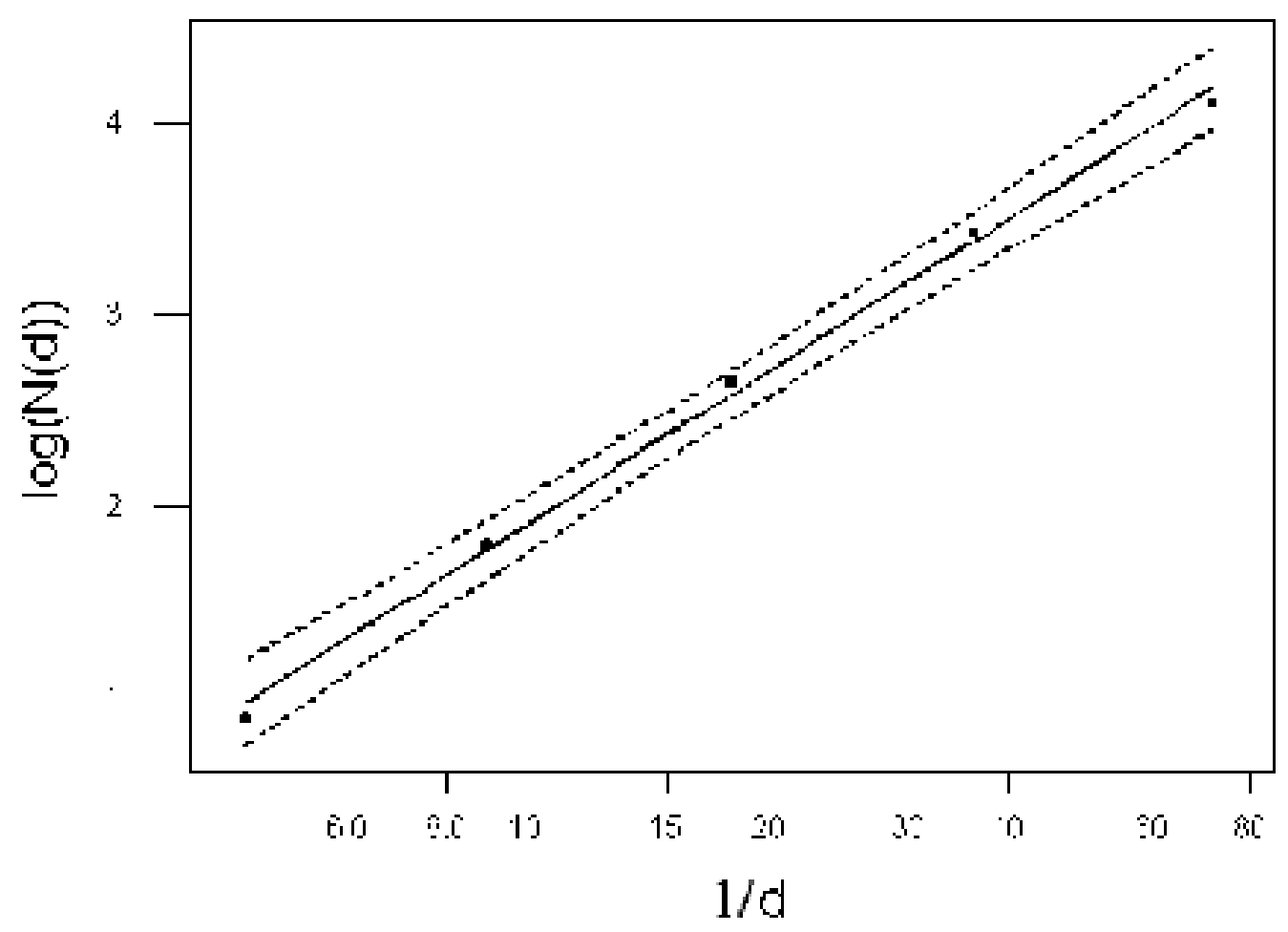

Figure 4: Box counting $\left(D_{0}\right)$ fit for SCN3 yielding $D_{0}=2.7(0.1)$. 

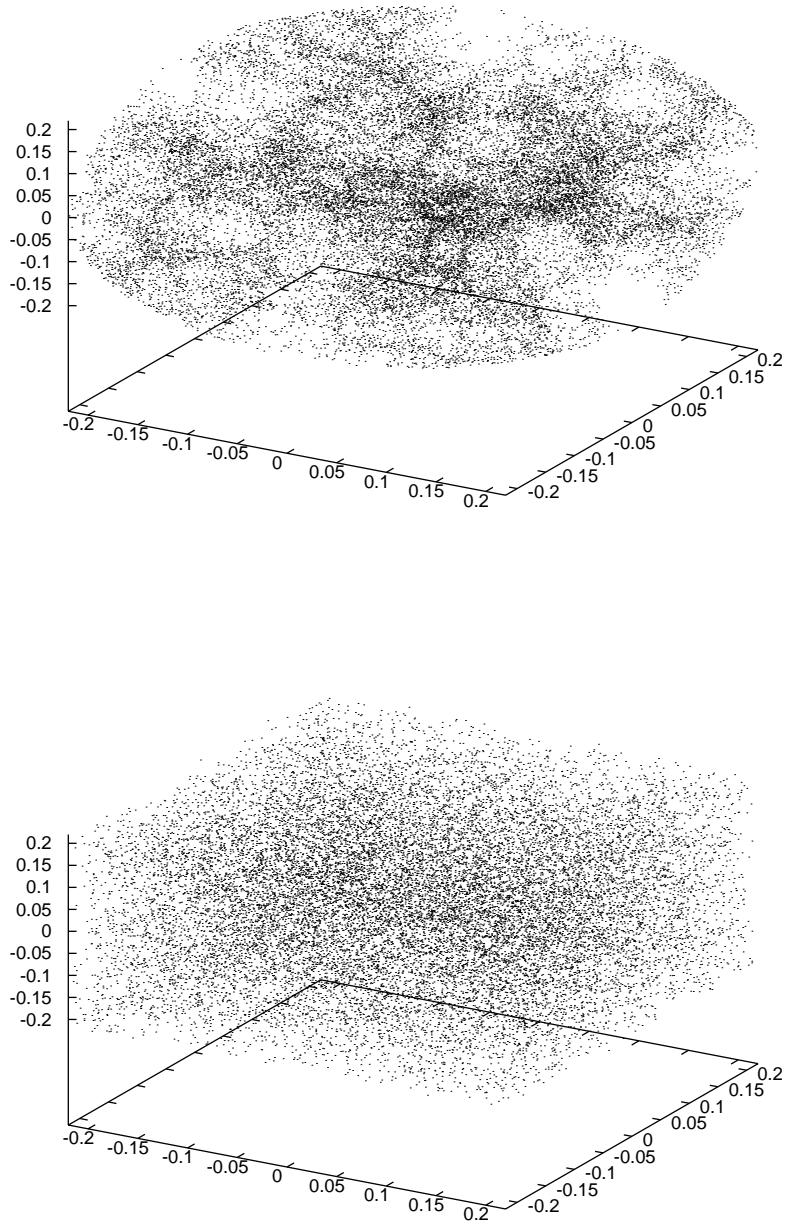

Figure 5: Three-dimensional point distributions, including: packing library (SCF3), randomly-populated $(x, y, z)$ coordinates $(\mathrm{RD})$. Each set possesses between 50000-60000 points. 

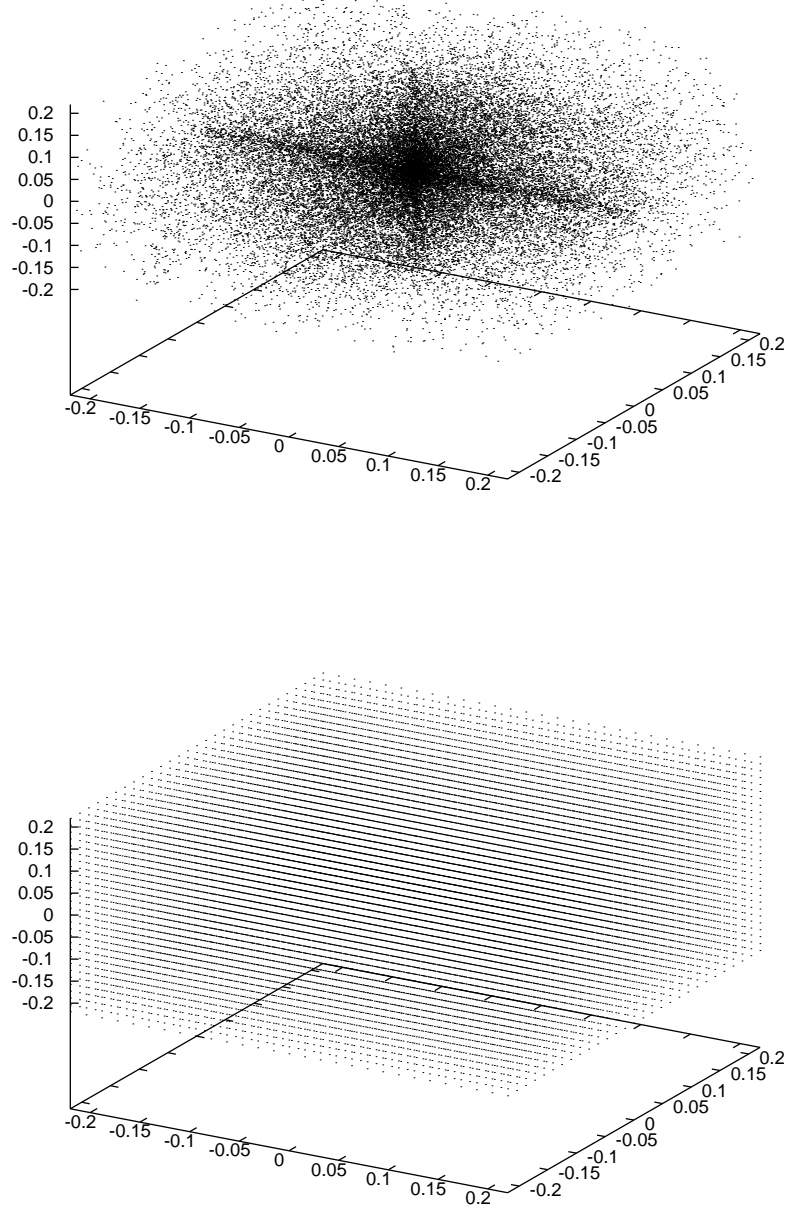

Figure 6: Three-dimensional point distributions, including linear-random (LR; top), and uniform distribution (UD; bottom). Each set possesses between 50000-60000 points. The LR set is comprised of randomly-populated $(r, \theta, \phi)$ points, in contrast to the random population of $(x, y, z)$ coordinates. 


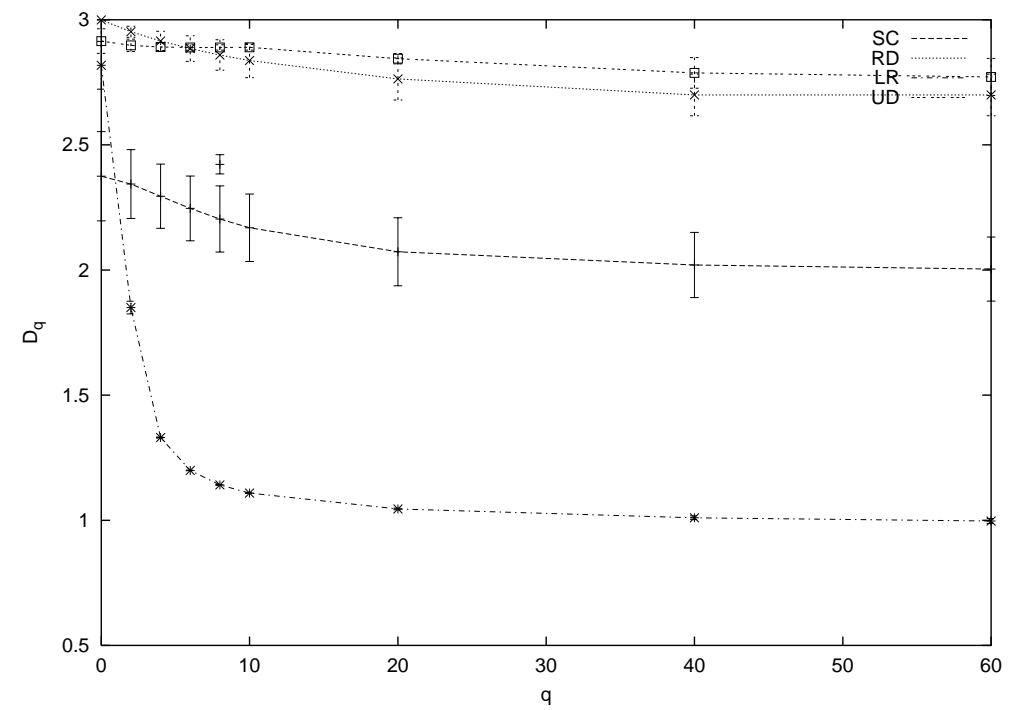

Figure 7: $D_{q}$ spectra $(q>0)$ for distributions of Figure [5] All sets show high $D_{0}$ values, with the RD and UD sets (top two curves) maintaining $D \sim 3$. Explicit structural differences can be seen in the SC and LR sets (bottom two curves) for large $q$, signifying strong inhomogeneous clustering behaviors. 


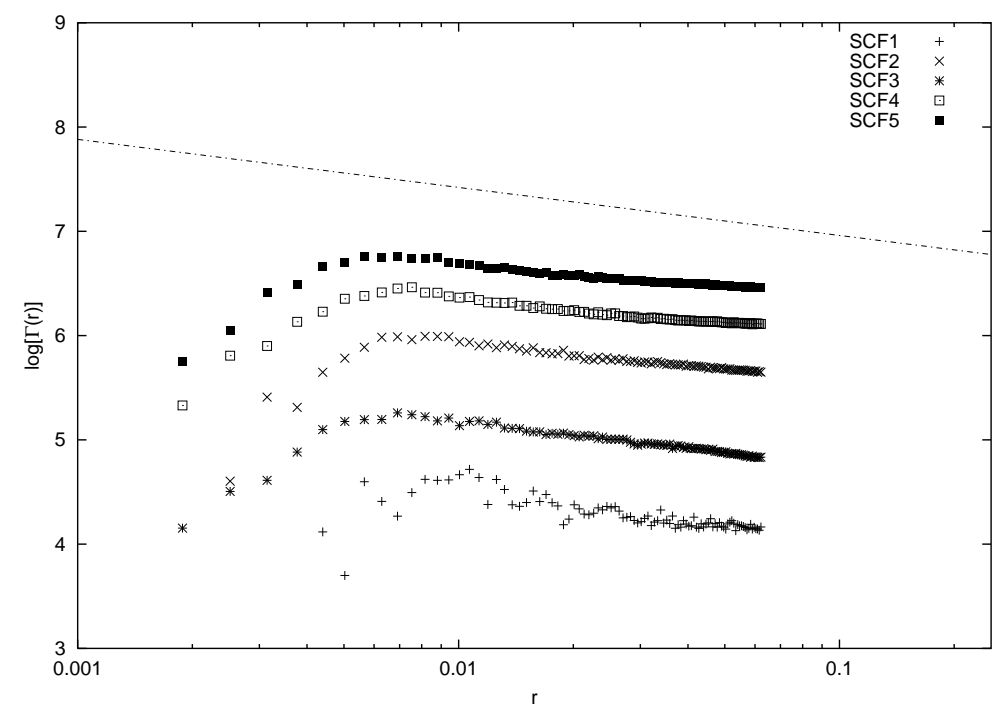

Figure 8: Conditional density $\Gamma(r)$ for flat libraries. Sample fit (dashed line) has slope $D-3=-0.2$, which implies $D=2.8$. Note that each library has been shifted vertically to eliminate overlap for easier viewing.

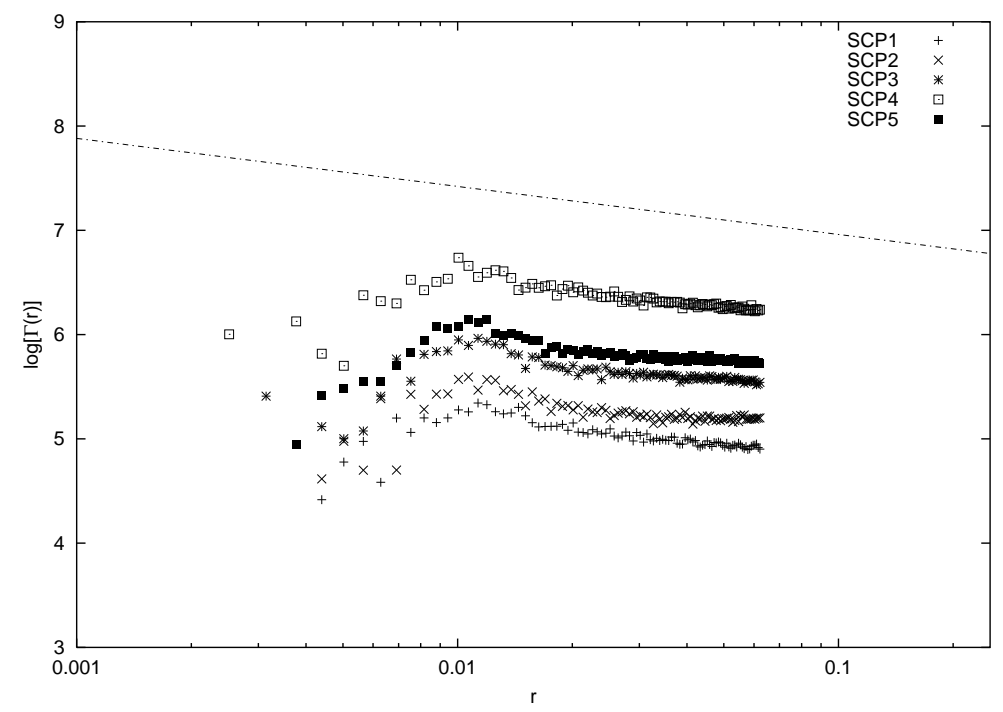

Figure 9: Conditional density $\Gamma(r)$ for positive libraries. Sample fit (dashed line) has slope $D-3=-0.2$, which implies $D=2.8$. 


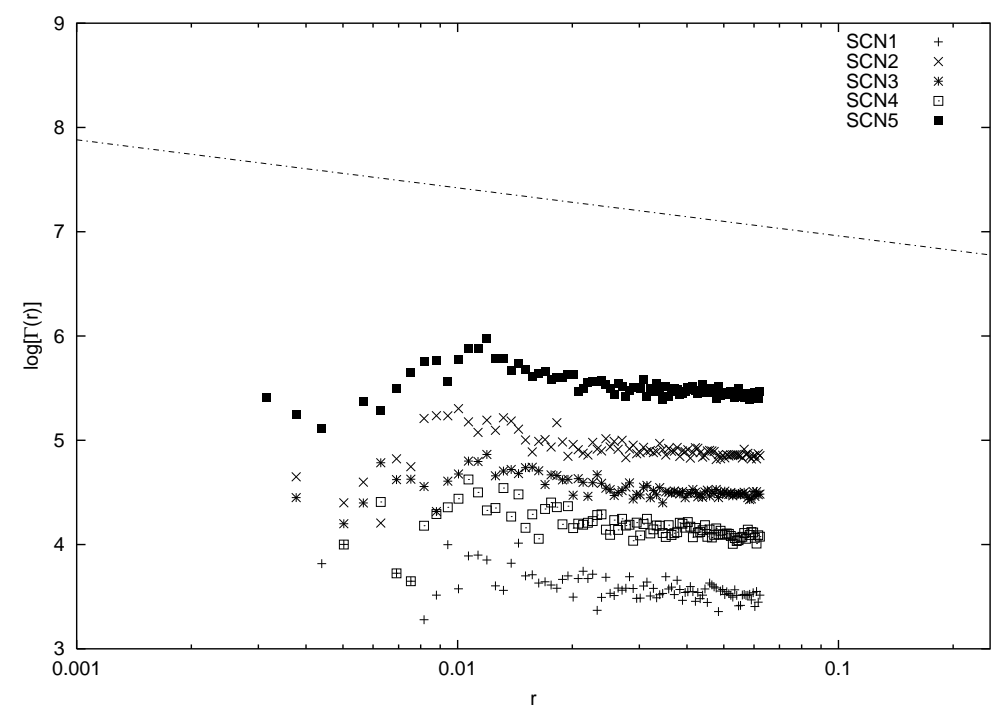

Figure 10: Conditional density $\Gamma(r)$ for negative libraries. Sample fit (dashed line) has slope $D-3=-0.2$, which implies $D=2.8$.

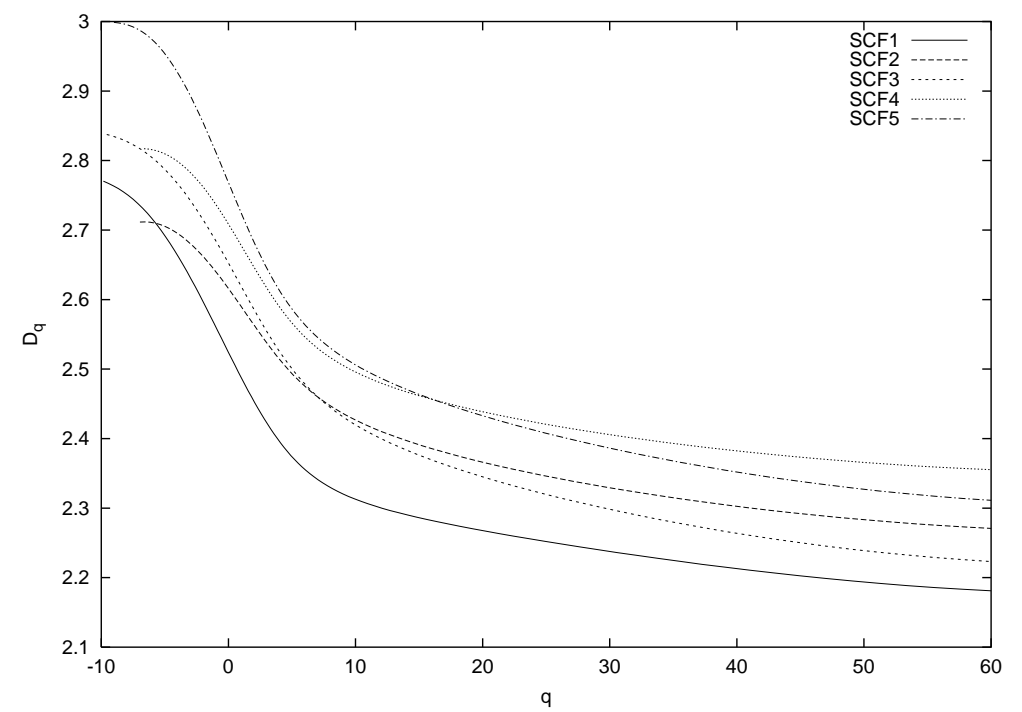

Figure 11: Bezier curve fit of full $D_{q}$ spectra for flat packing libraries, showing $D_{q} \rightarrow \sim 3$ for small $q . q<0$ values calculated by density reconstruction; $q \geq 0$ by box counting. 


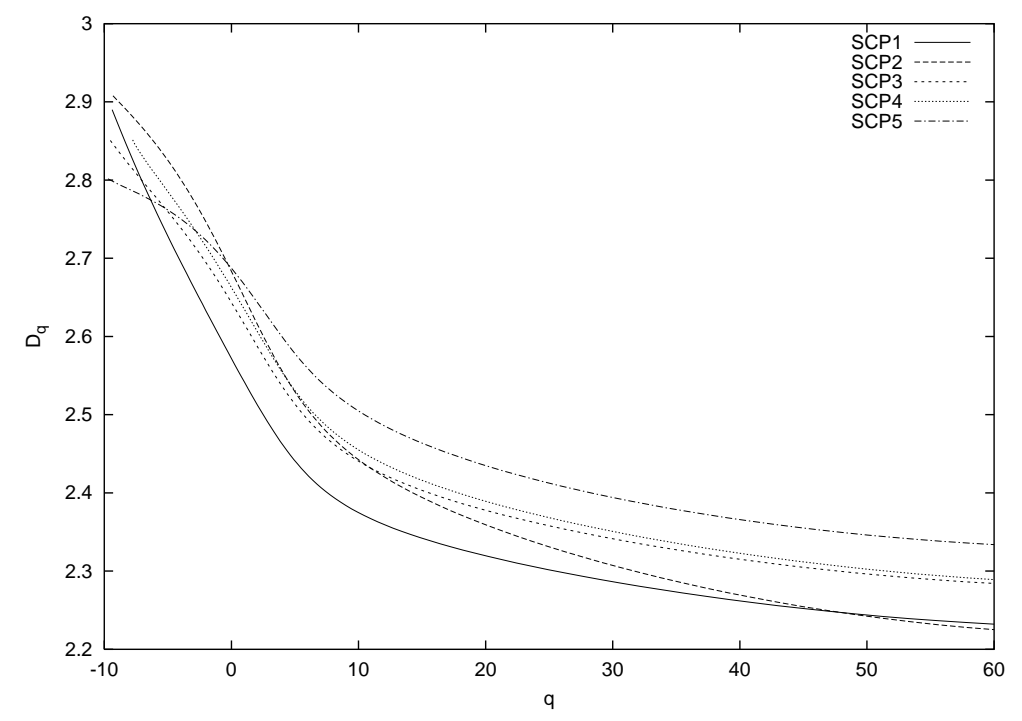

Figure 12: Bezier curve fit of full $D_{q}$ spectra for positive packing libraries.

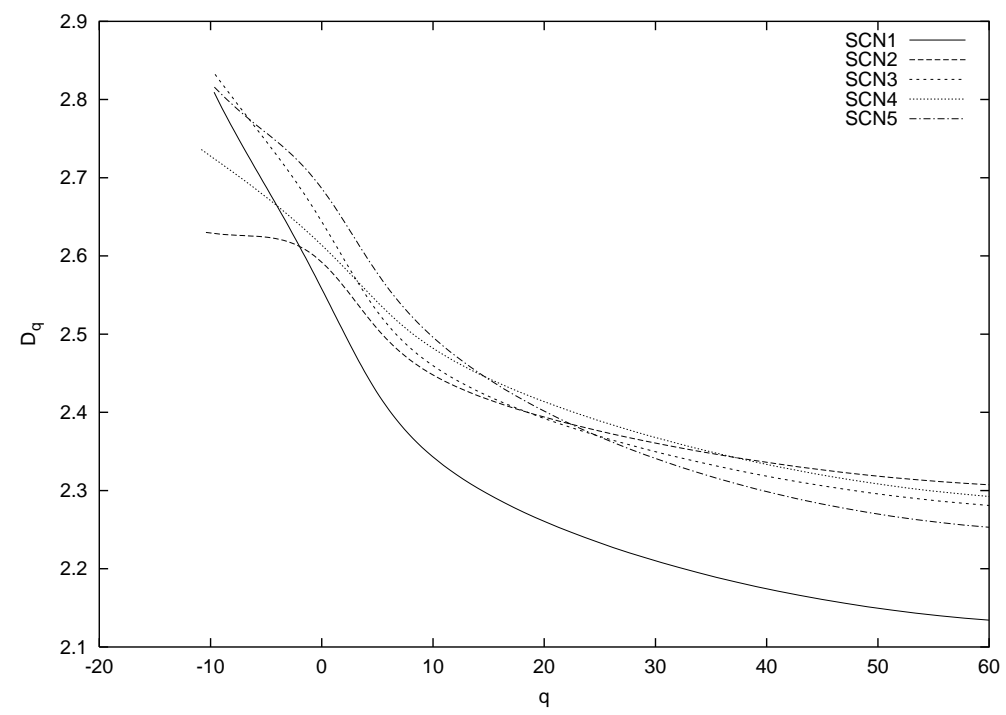

Figure 13: Bezier curve fit of full $D_{q}$ spectra for negative curvature packing libraries. 


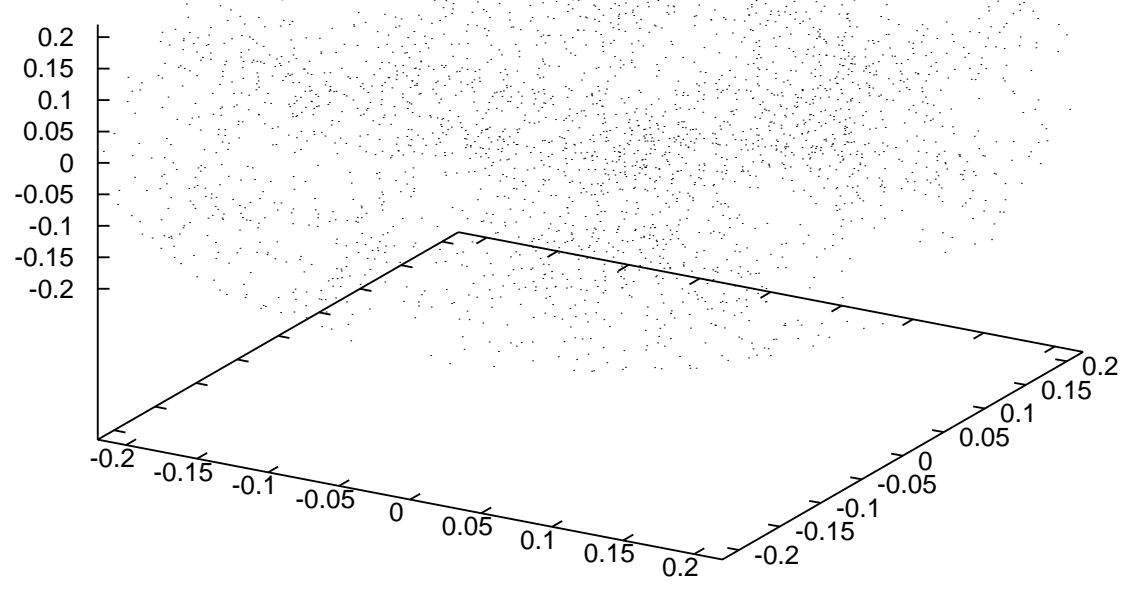

Figure 14: Library SCF3 with effective mass cutoff, containing $10 \%$ of spheres representing approximately $90 \%$ of total contained mass. 


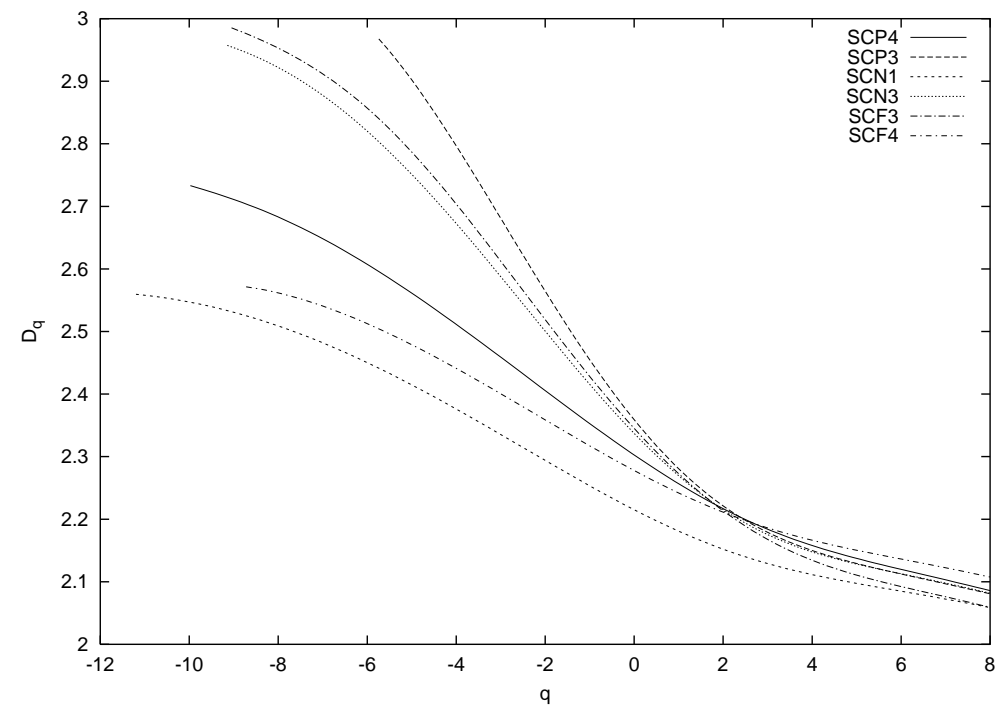

Figure 15: Bezier curve fit of full $D_{q}$ spectra for mass-reduced libraries, showing $D_{q} \rightarrow \sim 3$ for small $q . q<0$ values calculated by density reconstruction; $q \geq 0$ by box counting.

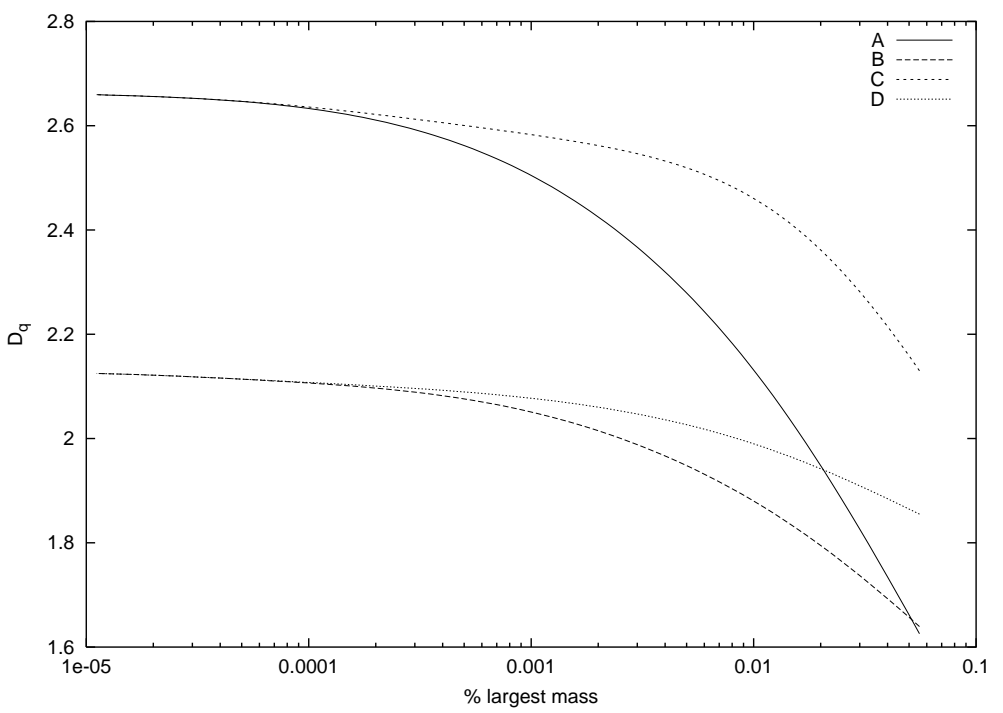

Figure 16: Variation of $D_{0}(\mathrm{~A}, \mathrm{C})$ and $D_{\infty}(\mathrm{B}, \mathrm{D})$ as a function of cutoff (\% of largest mass) for fixed number of box level iterations (A, B) and "lastpoint-removed" (C, D). True fractal dimension is bounded by the two curves. 


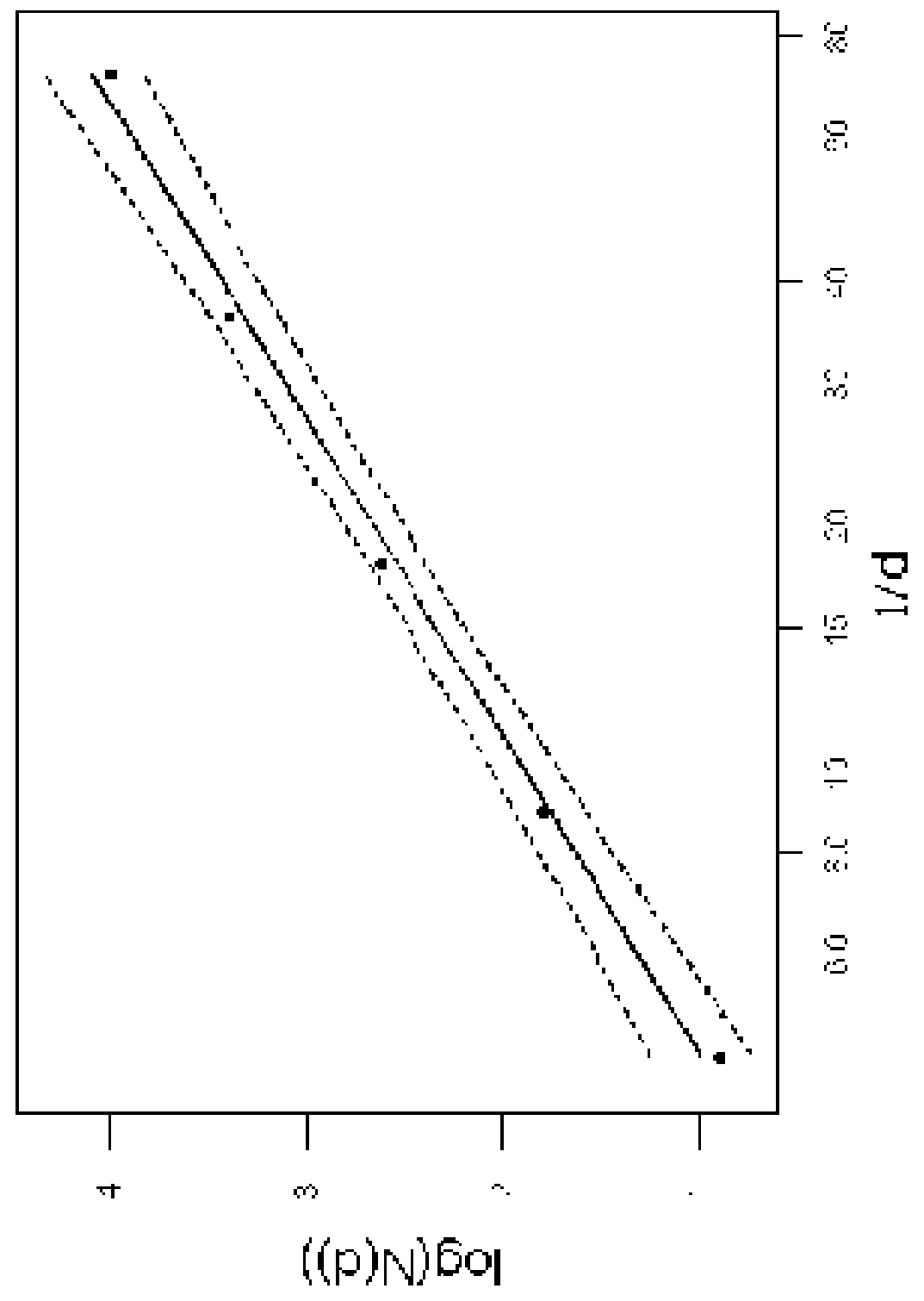




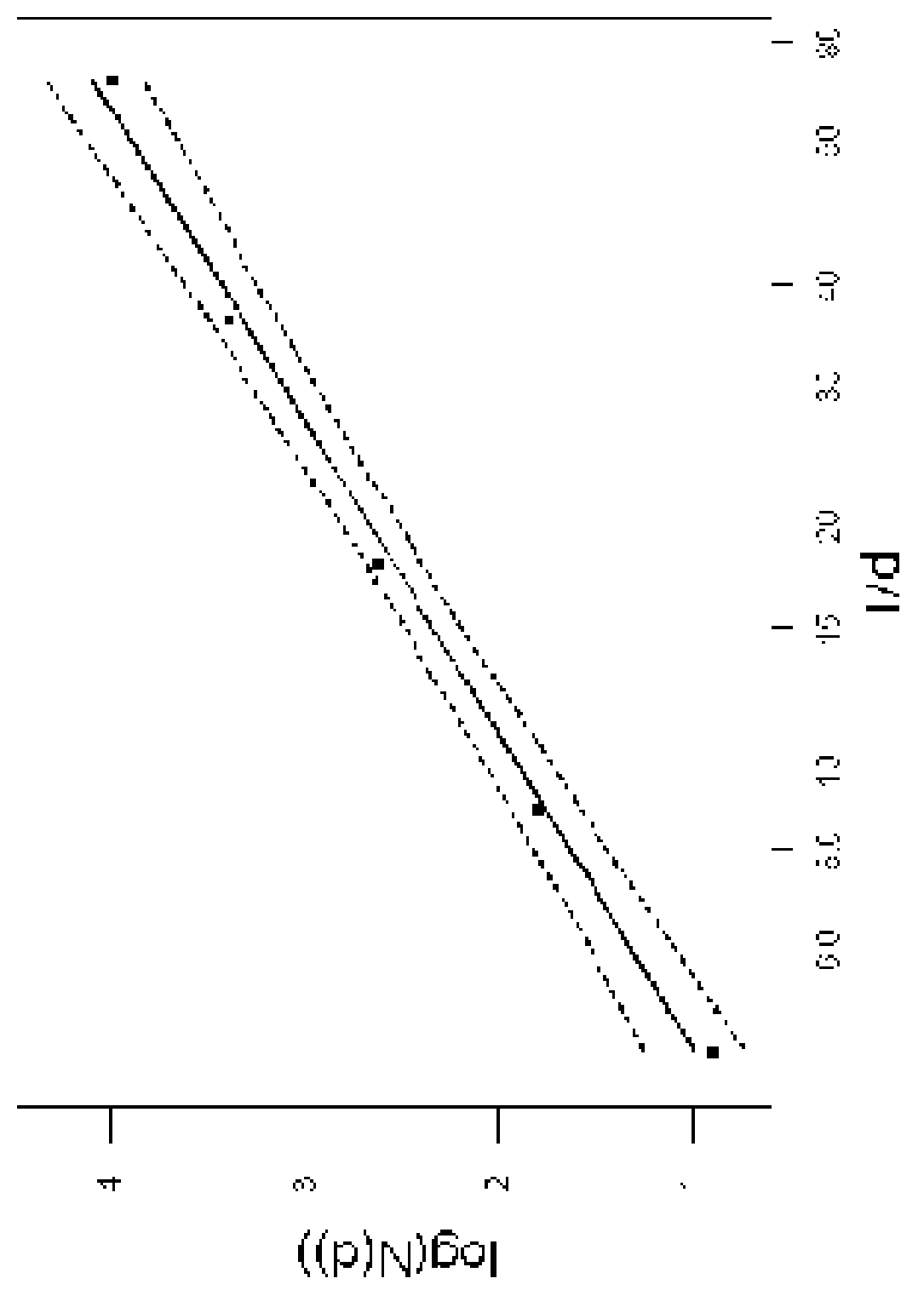

